Pacific

Journal of

Mathematics

\title{
BORNOLOGICAL QUANTUM GROUPS
}

Christian Voigt 


\title{
BORNOLOGICAL QUANTUM GROUPS
}

\author{
Christian VOIGT
}

\begin{abstract}
We introduce and study the concept of a bornological quantum group. This generalizes the theory of algebraic quantum groups in the sense of van Daele from the algebraic setting to the framework of bornological vector spaces. Working with bornological vector spaces allows to extend the scope of the latter theory considerably. In particular, the bornological theory covers smooth convolution algebras of arbitrary locally compact groups and their duals. Another source of examples arises from deformation quantization in the sense of Rieffel. Apart from describing these examples we obtain some general results on bornological quantum groups. In particular, we construct the dual of a bornological quantum group and prove the Pontrjagin duality theorem.
\end{abstract}

\section{Introduction}

The concept of a multiplier Hopf algebra introduced by van Daele [1994] extends the notion of a Hopf algebra to the setting of nonunital algebras. An important difference to the situation for ordinary Hopf algebras is that the comultiplication of a multiplier Hopf algebra $H$ takes values in the multiplier algebra $M(H \otimes H)$ and not in $H \otimes H$ itself. Due to the occurrence of multipliers, certain constructions with Hopf algebras have to be carried out more carefully in this context. Still, every multiplier Hopf algebra is equipped with a counit and an antipode satisfying analogues of the usual axioms. A basic example of a multiplier Hopf algebra is the algebra $C_{c}(\Gamma)$ of compactly supported functions on a discrete group $\Gamma$. This multiplier Hopf algebra is an ordinary Hopf algebra if and only if the group $\Gamma$ is finite.

Algebraic quantum groups form a special class of multiplier Hopf algebras with particularly nice properties. Every algebraic quantum group admits a dual quantum group and the analogue of the Pontrjagin duality theorem holds [van Daele 1998]. For instance, the multiplier Hopf algebra $C_{c}(\Gamma)$ associated to a discrete group $\Gamma$ is in fact an algebraic quantum group, its Pontrjagin dual being the complex group ring $\mathbb{C} \Gamma$. More generally, all discrete and all compact quantum groups can

MSC2000: 16W30, 81R50.

Keywords: multiplier Hopf algebras, quantum groups, bornological vector spaces. 
be viewed as algebraic quantum groups. In addition, the class of algebraic quantum groups is closed under some natural operations including the construction of the Drinfeld double [Drabant and van Daele 2001]. Moreover, algebraic quantum groups give rise to examples of locally compact quantum groups [Kustermans and van Daele 1997] illustrating nicely some general features of the latter.

However, due to the purely algebraic nature of the theory of multiplier Hopf algebras it is not possible to treat smooth convolution algebras of general Lie groups in this context, for instance. The theory of algebraic quantum groups only covers the case of locally compact groups having compact open subgroups [Landstad and van Daele 2007]. Accordingly, the variety of quantum groups that can be described in this setting is obviously limited. It is thus natural to look for a more general setup then the one provided by algebraic quantum groups.

Motivated by these facts we introduce in this paper the concept of a bornological quantum group. The main idea is to replace the category of vector spaces underlying the definition of an algebraic quantum group by the category of bornological vector spaces. It is worth pointing out that bornological vector spaces provide a natural setting for the study of various problems in noncommutative geometry and cyclic homology [Meyer 1999; 2004b; 2006, Voigt 2007].

Whereas the theory of locally convex vector spaces is based on the notion of an open subset, the key concept in the theory of bornological vector spaces is the notion of a bounded subset. It is clear from the definitions that both approaches are equivalent for normed spaces. In fact, bornological and topological analysis are essentially equivalent for Fréchet spaces [Meyer 2004a]. However, an important reason to prefer the bornological approach is that the category of bornological vector spaces has better algebraic properties than the category of locally convex vector spaces.

For the general theory of bornological quantum groups we follow the work by van Daele in the algebraic case. Basically, many constructions have to be rephrased in a more abstract way. This should not be surprising, in fact, the crucial feature of our definition of a bornological quantum group is that it allows us to prove important general results while being quite simple at the same time. Although stronger assertions are possible at several points of the paper our setup seems to be sufficiently general for most purposes.

It is natural to ask for the relation between our theory and the operator algebra approach to quantum groups. Although this will not be discussed in the present paper, let us include some remarks on this question. Under suitable additional assumptions, including the existence of a $*$-structure, a bornological quantum group can be completed to a locally compact quantum group in the sense of Kustermans and Vaes [Kustermans and Vaes 2000]. It is worth pointing out that the locally compact quantum groups arising in this way are always regular. Thus, from a 
slightly different perspective, nonregularity can be viewed as an obstruction to finding particularly nice subalgebras of "smooth functions" for locally compact quantum groups. This observation might be relevant in the context of the quantum E(2)-group [Woronowicz and Pusz 1999].

We are not aware of an example of a regular locally compact quantum group that cannot be obtained by the procedure above. On the other hand, it is clear from the examples in Section 10 that different bornological quantum groups may lead to the same locally compact quantum group.

\section{Bornological vector spaces}

In this section we review basic facts from the theory of bornological vector spaces. More information can be found in [Hogbe-Nlend 1970; 1977; Meyer 1999; 2004a]. Throughout we work over the complex numbers.

A bornological vector space is a vector space $V$ together with a collection $\mathfrak{S}(V)$ of subsets of $V$ satisfying certain conditions. These conditions can be viewed as an abstract reformulation of the properties of bounded subsets in a locally convex vector space. Following [Meyer 1999], we call a subset $S$ of a bornological vector space $V$ small if and only if it is contained in the bornology $\mathfrak{S}(V)$. Throughout the paper we only consider bornological vector spaces that are convex and complete.

The guiding example of a bornology is the collection of bounded subsets of a locally convex vector space. We write $\mathfrak{B o u n d}(V)$ for the bornological vector space associated to a locally convex vector space $V$ in this way. One obtains another bornological vector space $\mathfrak{C o m p}(V)$ by considering all precompact subsets of $V$ instead. In certain situations the precompact bornology has nicer properties than the bounded bornology. Finally, one may view an arbitrary vector space $V$ as a bornological vector space by considering the fine bornology $\mathfrak{F i n e}(V)$ consisting of the bounded subsets of finite dimensional subspaces of $V$.

A linear map $f: V \rightarrow W$ between bornological vector spaces is called bounded if it maps small subsets to small subsets. The space of bounded linear maps from $V$ to $W$ is denoted by $\operatorname{Hom}(V, W)$ and carries a natural bornology. In contrast, in the setting of locally convex vector spaces there are many different topologies on spaces of continuous linear maps.

We point out that the Hahn-Banach theorem does not hold for bornological vector spaces. The dual space $V^{\prime}$ of bounded linear functionals on a bornological vector space $V$ might very well be zero. A bornological vector space $V$ is called regular if the bounded linear functionals on $V$ separate points. The regularity of the underlying bornological vector space of a bornological quantum group will be guaranteed by the faithfulness of the Haar functional. All examples of bornological vector spaces arising from locally convex vector spaces are regular. 
Every complete bornological vector space can be written in a canonical way as a direct limit of Banach spaces. In this way analysis in bornological vector spaces reduces to analysis in Banach spaces.

There exists a natural tensor product in the category of complete bornological vector spaces. More precisely, the completed bornological tensor product $V \hat{\otimes} W$ is characterized by the universal property that bounded bilinear maps $V \times W \rightarrow X$ correspond to bounded linear maps $V \hat{\otimes} W \rightarrow X$. The bornological tensor product is associative and commutative and there is a natural adjunction isomorphism

$$
\operatorname{Hom}(V \hat{\otimes} W, X) \cong \operatorname{Hom}(V, \operatorname{Hom}(W, X))
$$

for all bornological vector spaces $V, W, X$. This relation is one of the main reasons that the category of bornological vector spaces is much better adapted for algebraic constructions than the category of locally convex spaces. Note that the completed projective tensor product in the category of locally convex spaces does not have a right adjoint functor because it does not commute with direct sums.

Throughout the paper we will use the leg numbering convention for maps defined on tensor products. Moreover, we sometimes write $\mathrm{id}_{(n)}$ to indicate that we consider the identity map on an $n$-fold tensor product.

A bornological algebra is a complete bornological vector space $A$ with an associative multiplication given as a bounded linear map $\mu: A \hat{\otimes} A \rightarrow A$. Remark that bornological algebras are not assumed to have a unit. Modules over bornological algebras and their homomorphisms are defined in the obvious way.

It follows immediately from the definitions that all linear maps $f: V \rightarrow W$ from a fine space $V$ into any bornological vector space $W$ are bounded. In particular there is a fully faithful functor $\mathfrak{F i n e}$ from the category of complex vector spaces into the category of bornological vector spaces. This embedding is compatible with tensor products. If $V_{1}$ and $V_{2}$ are fine spaces the completed bornological tensor product $V_{1} \hat{\otimes} V_{2}$ is the algebraic tensor product $V_{1} \otimes V_{2}$ equipped with the fine bornology. In particular, every algebra $A$ over the complex numbers can be viewed as a bornological algebra with the fine bornology.

In the case of Fréchet spaces a linear map $f: V \rightarrow W$ is bounded for the bounded or the precompact bornologies if and only if it is continuous. Hence the functors $\mathfrak{B o u n d}$ and $\mathfrak{C o m p}$ from the category of Fréchet spaces into the category of bornological vector spaces are fully faithful. The bornological tensor product of Fréchet spaces $V, W$ with the precompact bornology can be identified with the projective tensor product $V \hat{\otimes}_{\pi} W$ equipped with the precompact bornology, see [Meyer 1999].

In our considerations we will need the approximation property in order to avoid certain analytical problems with completed tensor products. We refer to [Meyer 2004a] for detailed information. 
Definition 2.1. Let $V$ be a complete bornological vector space. Then $V$ has the approximation property if the identity map of $V$ can be approximated uniformly on compact subsets by finite rank operators.

If $V$ is a Fréchet space, Grothendieck's approximation property for $V$ is equivalent to the bornological approximation property for $\mathfrak{C o m p}(V)$ [Meyer 2004a].

We will use the following two properties of bornological vector spaces satisfying the approximation property.

Lemma 2.2. Let $H$ be a bornological vector space satisfying the approximation property and let $\iota: V \rightarrow W$ be an injective bounded linear map. Then the induced bounded linear map id $\hat{\otimes} \iota: H \hat{\otimes} V \rightarrow H \hat{\otimes} W$ is injective as well.

Lemma 2.3. Let $H$ be a bornological vector space satisfying the approximation property and let $V$ be an arbitrary bornological vector space. Then the canonical linear map $\iota: H \hat{\otimes} V^{\prime} \rightarrow \operatorname{Hom}(V, H)$ is injective.

\section{Multiplier algebras}

In this section we prove basic results on multiplier algebras of bornological algebras that will be needed in the sequel.

The theory of multiplier Hopf algebras is an extension of the theory of Hopf algebras to the case where the underlying algebras do not have an identity element. Similarly, in our setting we will have to work with nonunital bornological algebras. We will work with bornological algebras that are essential in the following sense.

Definition 3.1. A bornological algebra $H$ is called essential if the multiplication map induces an isomorphism $H \hat{\otimes}_{H} H \cong H$.

In order to avoid trivialities we shall always assume that essential bornological algebras are different from zero. Clearly, every unital bornological algebra is essential. If $H$ has an approximate identity [Meyer 2004b] then $H$ is essential if and only if the multiplication $H \hat{\otimes} H \rightarrow H$ is a bornological quotient map. We will not require the existence of approximate identities in the general definition of a bornological quantum group.

Definition 3.2. Let $H$ be a bornological algebra. An $H$-module $V$ is called essential if the canonical map $H \hat{\otimes}_{H} V \rightarrow V$ is an isomorphism.

An analogous definition can be given for right modules. In particular, an essential algebra $H$ is an essential left and right module over itself.

We shall now discuss multipliers. A left multiplier for a bornological algebra $H$ is a bounded linear map $L: H \rightarrow H$ such that $L(f g)=L(f) g$ for all $f, g \in$ $H$. Similarly, a right multiplier is a bounded linear map $R: H \rightarrow H$ such that $R(f g)=f R(g)$ for all $f, g \in H$. We let $M_{l}(H)$ and $M_{r}(H)$ be the spaces of left 
and right multipliers, respectively. These spaces are equipped with the subspace bornology of $\operatorname{Hom}(H, H)$ and become bornological algebras with multiplication given by composition of maps. The multiplier algebra $M(H)$ of a bornological algebra $H$ is the space of all pairs $(L, R)$ where $L$ is a left multiplier and $R$ is a right multiplier for $H$ such that $f L(g)=R(f) g$ for all $f, g \in H$. The bornology and algebra structure of $M(H)$ are inherited from $M_{l}(H) \oplus M_{r}(H)$. There is a natural homomorphism $\iota: H \rightarrow M(H)$. By construction, $H$ is a left and right $M(H)$-module.

Let $H$ and $K$ be bornological algebras and let $f: H \rightarrow M(K)$ be a homomorphism. Then $K$ is a left and right $H$-module in an obvious way. We say that the homomorphism $f: H \rightarrow M(K)$ is essential if it turns $K$ into an essential left and right $H$-module. That is, for the corresponding module structures we have $H \hat{\otimes}_{H} K \cong K \cong K \hat{\otimes}_{H} H$ in this case. Note that the identity map id : $H \rightarrow H$ defines an essential homomorphism $H \rightarrow M(H)$ if and only if the bornological algebra $H$ is essential.

Lemma 3.3. Let $H$ be a bornological algebra and let $f: H \rightarrow M(K)$ be an essential homomorphism into the multiplier algebra of an essential bornological algebra $K$. Then there exists a unique unital homomorphism $F: M(H) \rightarrow M(K)$ such that $F \iota=f$ where $\iota: H \rightarrow M(H)$ is the canonical map.

Proof. We obtain a bounded linear map $F_{l}: M_{l}(H) \rightarrow M_{l}(K)$ by

$$
M_{l}(H) \hat{\otimes} K \cong M_{l}(H) \hat{\otimes} H \hat{\otimes}_{H} K \stackrel{\mu \hat{\otimes} \mathrm{id}}{\longrightarrow} H \hat{\otimes}_{H} K \cong K
$$

and accordingly a map $F_{r}: M_{r}(H) \rightarrow M_{r}(K)$ by

$$
K \hat{\otimes} M_{r}(H) \cong K \hat{\otimes}_{H} H \hat{\otimes} M_{r}(H) \stackrel{\operatorname{id} \hat{\otimes} \mu}{\longrightarrow} K \hat{\otimes}_{H} H \cong K .
$$

It is straightforward to check that $F((L, R))=\left(F_{l}(L), F_{r}(R)\right)$ defines a unital homomorphism $F: M(H) \rightarrow M(K)$ such that $F \iota=f$. Uniqueness of $F$ follows from the fact that $f(H) \cdot K \subset K$ and $K \cdot f(H) \subset K$ are dense subspaces.

The next result, on tensor products, is easily proved.

Lemma 3.4. Let $H_{1}, H_{2}$ be essential bornological algebras and let $f_{1}: H_{1} \rightarrow$ $M\left(K_{1}\right)$ and $f_{2}: H_{2} \rightarrow M\left(K_{2}\right)$ be essential homomorphisms into the multiplier algebras of bornological algebras $K_{1}$ and $K_{2}$. Then the induced homomorphism $f_{1} \hat{\otimes} f_{2}: H_{1} \hat{\otimes} H_{2} \rightarrow M\left(K_{1} \hat{\otimes} K_{2}\right)$ is essential.

Following [van Daele 1994], we say that a bornological algebra $H$ is nondegenerate if $f g=0$ for all $g \in H$ implies $f=0$ and $f g=0$ for all $f$ implies $g=0$. These conditions are equivalent to saying that the natural maps

$$
H \rightarrow M_{l}(H), \quad H \rightarrow M_{r}(H)
$$


are injective. In particular, for a nondegenerate bornological algebra the canonical map $H \rightarrow M(H)$ is injective.

Nondegeneracy of a bornological algebra is a consequence of the existence of a faithful linear functional in the following sense.

Definition 3.5. Let $H$ be a bornological algebra. A bounded linear functional $\omega: H \rightarrow \mathbb{C}$ is called faithful if $\omega(f g)=0$ for all $g$ implies $f=0$ and $\omega(f g)=0$ for all $f$ implies $g=0$.

Remark that a bornological algebra $H$ equipped with a faithful bounded linear functional is regular in the sense that bounded linear functionals separate the points of $H$.

Lemma 3.6. Let $H_{1}$ and $H_{2}$ be bornological algebras satisfying the approximation property equipped with faithful bounded linear functionals $\phi_{1}$ and $\phi_{2}$, respectively. Then $\phi_{1} \hat{\otimes} \phi_{2}$ is a faithful linear functional on $H_{1} \hat{\otimes} H_{2}$.

Proof. Assume that $x \in H_{1} \hat{\otimes} H_{2}$ satisfies $\left(\phi_{1} \hat{\otimes} \phi_{2}\right)(x y)=0$ for all $y \in H_{1} \hat{\otimes} H_{2}$. Since $\phi_{1}$ is faithful the bounded linear map $\mathscr{F}_{1}: H_{1} \rightarrow H_{1}^{\prime}$ given by $\mathscr{F}_{1}(f)(g)=$ $\phi_{1}(f g)$ is injective. Similarly, we have an injective bounded linear map $\mathscr{F}_{2}: H_{2} \rightarrow$ $H_{2}^{\prime}$ given by $\mathscr{F}_{2}(f)(g)=\phi_{2}(f g)$. Consider the composition

$$
H_{1} \hat{\otimes} H_{2} \longrightarrow H_{1}^{\prime} \hat{\otimes} H_{2} \longrightarrow \operatorname{Hom}\left(H_{1}, H_{2}\right) \longrightarrow \operatorname{Hom}\left(H_{1}, H_{2}^{\prime}\right),
$$

where the first and third map are induced by $\mathscr{F}_{1}$ and $\mathscr{F}_{2}$, respectively, and the second arrow is the obvious one. The first of these maps is injective according to Lemma 2.2, the second map is injective according to Lemma 2.3, and injectivity of the third map is obvious. According to our assumption we see that the image of $x$ in $\operatorname{Hom}\left(H_{1}, H_{2}^{\prime}\right)$ is zero. Consequently we have $x=0$. In a similar way one shows that $\left(\phi_{1} \hat{\otimes} \phi_{2}\right)(y x)=0$ for all $y \in H_{1} \hat{\otimes} H_{2}$ implies $x=0$.

\section{Bornological quantum groups}

In this section we introduce the notion of a bornological quantum group. Moreover we prove that every bornological quantum group is equipped with a counit and an invertible antipode.

In the sequel we assume that $H$ is an essential bornological algebra satisfying the approximation property. Moreover we suppose that $H$ is equipped with a faithful bounded linear functional. Remark that we may thus view $H$ as a subset of the multiplier algebra $M(H)$. Taking into account Lemma 3.6, an analogous statement applies to tensor powers of $H$.

First we have to discuss the concept of a comultiplication on $H$. Let $\Delta: H \rightarrow$ $M(H \hat{\otimes} H)$ be a homomorphism. The left Galois maps $\gamma_{l}, \gamma_{r}: H \hat{\otimes} H \rightarrow M(H \hat{\otimes} H)$ 
for $\Delta$ are defined by

$$
\gamma_{l}(f \otimes g)=\Delta(f)(g \otimes 1), \quad \gamma_{r}(f \otimes g)=\Delta(f)(1 \otimes g) .
$$

Similarly, the right Galois maps $\rho_{l}, \rho_{r}: H \hat{\otimes} H \rightarrow M(H \hat{\otimes} H)$ for $\Delta$ are defined by

$$
\rho_{l}(f \otimes g)=(f \otimes 1) \Delta(g), \quad \rho_{r}(f \otimes g)=(1 \otimes f) \Delta(g) .
$$

The appropriate analogues of these maps play an important role in the algebraic as well as the analytic theory of quantum groups [van Daele 1994; 1998, Baaj and Skandalis 1993]. In the operator algebra approach to quantum groups they correspond to multiplicative unitaries [Baaj and Skandalis 1993]. Our terminology is motivated from the fact that variants of these maps also occur in the theory of Hopf-Galois extensions; see for instance [Montgomery 1993].

Assume in addition that the homomorphism $\Delta: H \rightarrow M(H \hat{\otimes} H)$ is essential. Then $\Delta$ is called coassociative if

$$
(\Delta \hat{\otimes} \mathrm{id}) \Delta=(\mathrm{id} \hat{\otimes} \Delta) \Delta,
$$

where both sides are viewed as maps from $H$ to $M(H \hat{\otimes} H \hat{\otimes} H)$. These maps are well-defined according to Lemma 3.4.

Definition 4.1. An essential homomorphism $\Delta: H \rightarrow M(H \hat{\otimes} H)$ is called a comultiplication if it is coassociative.

An essential algebra homomorphism $f: H \rightarrow M(K)$ between bornological algebras equipped with comultiplications is called a coalgebra homomorphism if $\Delta f=(f \hat{\otimes} f) \Delta$.

We need some more terminology. The opposite algebra $H^{\text {op }}$ of $H$ is the space $H$ equipped with the opposite multiplication. That is, the multiplication $\mu^{\mathrm{op}}$ in $H^{\mathrm{op}}$ is defined by $\mu^{\mathrm{op}}=\mu \tau$ where $\mu: H \hat{\otimes} H \rightarrow H$ is the multiplication in $H$ and $\tau: H \hat{\otimes} H \rightarrow H \hat{\otimes} H$ is the flip map given by $\tau(f \otimes g)=g \otimes f$. An algebra antihomomorphism between $H$ and $K$ is an algebra homomorphism $\phi: H \rightarrow$ $K^{\mathrm{op}}$. Equivalently, an algebra antihomomorphism can be viewed as an algebra homomorphism $H^{\text {op }} \rightarrow K$. If $\Delta: H \rightarrow M(H \hat{\otimes} H)$ is a comultiplication then $\Delta$ also defines a comultiplication $H^{\mathrm{op}} \rightarrow M\left(H^{\mathrm{op}} \hat{\otimes} H^{\mathrm{op}}\right)$. We write $\gamma_{l}^{\mathrm{op}}, \gamma_{r}^{\mathrm{op}}, \rho_{l}^{\mathrm{op}}, \rho_{r}^{\mathrm{op}}$ for the corresponding Galois maps.

Apart from changing the order of multiplication we may also reverse the order of a comultiplication. If $\Delta: H \rightarrow M(H \hat{\otimes} H)$ is a comultiplication then the opposite comultiplication $\Delta^{\text {cop }}$ is the essential homomorphism from $H$ to $M(H \hat{\otimes} H)$ defined by $\Delta^{\text {cop }}=\tau \Delta$. We write $\gamma_{l}^{\text {cop }}, \gamma_{r}^{\text {cop }}, \rho_{l}^{\text {cop }}, \rho_{r}^{\text {cop }}$ for the Galois maps associated to this comultiplication. Moreover we write $H^{\text {cop }}$ for $H$ equipped with the opposite comultiplication. Using opposite comultiplications we obtain the notion of a coalgebra antihomomorphism. 
We may also combine these procedures, that is, reverse both multiplication and comultiplication. The bornological algebra with comultiplication arising in this way is denoted by $H^{\text {op cop }}=\left(H^{\text {op }}\right)^{\text {cop }}$ and we write $\gamma_{l}^{\text {op cop }}, \gamma_{r}^{\text {op cop }}, \rho_{l}^{\text {op cop }}, \rho_{r}^{\text {op cop }}$ for the corresponding Galois maps.

It is straightforward to check that the Galois maps of $H, H^{\mathrm{op}}, H^{\text {cop }}$ and $H^{\mathrm{opcop}}$ are related as follows.

Lemma 4.2. Let $\Delta: H \rightarrow M(H \hat{\otimes} H)$ be a comultiplication. Then

$$
\begin{array}{lll}
\gamma_{r}=\tau \gamma_{l}^{\mathrm{cop},} & \rho_{l}=\gamma_{l}^{\mathrm{op}} \tau, & \rho_{r}=\tau \gamma_{l}^{\mathrm{op} \mathrm{cop}} \tau, \\
\gamma_{l}=\tau \gamma_{r}^{\mathrm{cop},} & \rho_{r}=\gamma_{r}^{\mathrm{op}} \tau, & \rho_{l}=\tau \gamma_{r}^{\mathrm{op} \mathrm{cop}} \tau, \\
\rho_{r}=\tau \rho_{l}^{\mathrm{cop}}, & \gamma_{l}=\rho_{l}^{\mathrm{op}} \tau, & \gamma_{r}=\tau \rho_{l}^{\mathrm{opcop}} \tau, \\
\rho_{l}=\tau \rho_{r}^{\mathrm{cop}}, & \gamma_{r}=\rho_{r}^{\mathrm{op}} \tau, & \gamma_{l}=\tau \rho_{r}^{\mathrm{opcop}} \tau
\end{array}
$$

for the Galois maps of $H, H^{\mathrm{op}}, H^{\mathrm{cop}}$ and $H^{\mathrm{op} \text { cop }}$.

These relations can also be rewritten in the form

$$
\begin{array}{lll}
\gamma_{l}^{\mathrm{cop}}=\tau \gamma_{r}, & \gamma_{l}^{\mathrm{op}}=\rho_{l} \tau, & \gamma_{l}^{\mathrm{opcop}}=\tau \rho_{r} \tau, \\
\gamma_{r}^{\mathrm{cop}}=\tau \gamma_{l}, & \gamma_{r}^{\mathrm{op}}=\rho_{r} \tau, & \gamma_{r}^{\mathrm{op} \mathrm{cop}}=\tau \rho_{l} \tau, \\
\rho_{l}^{\mathrm{cop}}=\tau \rho_{r}, & \rho_{l}^{\mathrm{op}}=\gamma_{l} \tau, & \rho_{l}^{\mathrm{opcop}}=\tau \gamma_{r} \tau, \\
\rho_{r}^{\mathrm{cop}}=\tau \rho_{l}, & \rho_{r}^{\mathrm{op}}=\gamma_{r} \tau, & \rho_{r}^{\mathrm{op} \mathrm{cop}}=\tau \gamma_{l} \tau .
\end{array}
$$

As a consequence, the Galois maps for $H$ may be expressed in terms of the maps $\gamma_{l}, \gamma_{l}^{\text {op }}, \gamma_{l}^{\text {cop }}$ and $\gamma_{l}^{\text {op cop }}$ and vice versa. Of course, there are similar statements for $\gamma_{r}, \rho_{l}$ and $\rho_{r}$. This basic observation will be used frequently below.

Let $\Delta: H \rightarrow M(H \hat{\otimes} H)$ be a comultiplication such that all Galois maps associated to $\Delta$ define bounded linear maps from $H \hat{\otimes} H$ into itself. If $\omega$ is a bounded linear functional on $H$ we define for every $f \in H$ a multiplier (id $\hat{\otimes} \omega) \Delta(f) \in M(H)$ by

$$
\begin{aligned}
& (\operatorname{id} \hat{\otimes} \omega) \Delta(f) \cdot g=(\operatorname{id} \hat{\otimes} \omega) \gamma_{l}(f \otimes g) \\
& g \cdot(\operatorname{id} \hat{\otimes} \omega) \Delta(f)=(\operatorname{id} \hat{\otimes} \omega) \rho_{l}(g \otimes f) .
\end{aligned}
$$

To check that this is indeed a two-sided multiplier observe that

$$
(f \otimes 1) \gamma_{l}(g \otimes h)=\rho_{l}(f \otimes g)(h \otimes 1)
$$

for all $f, g, h \in H$. In a similar way we define $(\omega \hat{\otimes} \mathrm{id}) \Delta(f) \in M(H)$ by

$$
\begin{aligned}
& (\omega \hat{\otimes} \mathrm{id}) \Delta(f) \cdot g=(\mathrm{id} \otimes \omega) \gamma_{r}(f \otimes g) \\
& g \cdot(\omega \hat{\otimes} \mathrm{id}) \Delta(f)=(\mathrm{id} \otimes \omega) \rho_{r}(g \otimes f) .
\end{aligned}
$$


Definition 4.3. Let $\Delta: H \rightarrow M(H \hat{\otimes} H)$ be a comultiplication such that all Galois maps associated to $\Delta$ define bounded linear maps from $H \hat{\otimes} H$ into itself.

A bounded linear functional $\phi: H \rightarrow \mathbb{C}$ is called left invariant if

$$
(\mathrm{id} \hat{\otimes} \phi) \Delta(f)=\phi(f) 1
$$

for all $f \in H$. Similarly, a bounded linear functional $\psi: H \rightarrow \mathbb{C}$ is called right invariant if

$$
(\psi \hat{\otimes} \mathrm{id}) \Delta(f)=\psi(f) 1
$$

for all $f \in H$.

We now give the definition of a bornological quantum group.

Definition 4.4. A bornological quantum group is an essential bornological algebra $H$ satisfying the approximation property together with a comultiplication $\Delta: H \rightarrow$ $M(H \hat{\otimes} H)$ such that all Galois maps associated to $\Delta$ are isomorphisms and a faithful left invariant functional $\phi: H \rightarrow \mathbb{C}$.

A morphism between bornological quantum groups $H$ and $K$ is an essential algebra homomorphism $\alpha: H \rightarrow M(K)$ such that $(\alpha \hat{\otimes} \alpha) \Delta=\Delta \alpha$.

To be precise, the Galois maps in a bornological quantum group are supposed to yield bornological isomorphisms of $H \hat{\otimes} H$ into itself. The left invariant functional $\phi$ is also referred to as the left Haar functional.

Our definition of a bornological quantum group is equivalent to the definition of an algebraic quantum group in the sense of van Daele [van Daele 1998] provided the underlying bornological vector space carries the fine bornology. The only difference in this case is that we have included faithfulness of the Haar functional in the definition.

An easy computation yields the following assertion.

Lemma 4.5. Let $H$ be a bornological quantum group. Then

$$
\left(\rho_{l} \hat{\otimes} \mathrm{id}\right)\left(\mathrm{id} \hat{\otimes} \gamma_{r}\right)=\left(\mathrm{id} \hat{\otimes} \gamma_{r}\right)\left(\rho_{l} \hat{\otimes} \mathrm{id}\right)
$$

where both sides are viewed as maps from $H \hat{\otimes} H \hat{\otimes} H$ into itself.

The next theorem provides an alternative description of bornological quantum groups.

Theorem 4.6. Let $H$ be an essential bornological algebra satisfying the approximation property and let $\Delta: H \rightarrow M(H \hat{\otimes} H)$ be a comultiplication such that all associated Galois maps define bounded linear maps from $H \hat{\otimes} H$ to itself. Moreover assume that $\phi: H \rightarrow \mathbb{C}$ is a faithful left invariant functional. Then $H$ is a bornological quantum group if and only if there exist an essential algebra 
homomorphism $\epsilon: H \rightarrow \mathbb{C}$ and a linear isomorphism $S: H \rightarrow H$ which is both an algebra antihomomorphism and a coalgebra antihomomorphism such that

$$
(\epsilon \hat{\otimes} \mathrm{id}) \Delta=\mathrm{id}=(\mathrm{id} \hat{\otimes} \epsilon) \Delta
$$

and

$$
\mu(S \hat{\otimes} \mathrm{id}) \gamma_{r}=\epsilon \hat{\otimes} \mathrm{id}, \quad \mu(\mathrm{id} \hat{\otimes} S) \rho_{l}=\mathrm{id} \hat{\otimes} \epsilon .
$$

In this case the maps $\epsilon$ and $S$ are uniquely determined.

Proof. The proof follows the discussion in [van Daele 1994]. Along the way we obtain some formulas which are also useful in other situations.

Let us first assume that there exist maps $\epsilon$ and $S$ satisfying the above conditions. Following the traditional terminology, these maps will be called the counit and the antipode of $H$. We claim that the inverse $\gamma_{r}^{-1}$ of $\gamma_{r}$ is given by

$$
\gamma_{r}^{-1}=\left(S^{-1} \hat{\otimes} \mathrm{id}\right) \gamma_{r}^{\mathrm{cop}}(S \hat{\otimes} \mathrm{id}) .
$$

Using that $S$ is a coalgebra antihomomorphism we obtain the equality

$$
\begin{gathered}
\left(S^{-1} \hat{\otimes} \mathrm{id}\right) \gamma_{r}^{\mathrm{cop}}(S \hat{\otimes} \mathrm{id})=\left(S^{-1} \hat{\otimes} \mathrm{id}\right)(\mathrm{id} \hat{\otimes} \mu)\left(\Delta^{\mathrm{cop}} \hat{\otimes} \mathrm{id}\right)(S \hat{\otimes} \mathrm{id}) \\
=(\mathrm{id} \hat{\otimes} \mu)\left(S^{-1} \hat{\otimes} \mathrm{id} \hat{\otimes} \mathrm{id}\right)(\tau \Delta S \hat{\otimes} \mathrm{id}) \\
=(\mathrm{id} \hat{\otimes} \mu)(\mathrm{id} \hat{\otimes} S \hat{\otimes} \mathrm{id})(\Delta \hat{\otimes} \mathrm{id}),
\end{gathered}
$$

where both sides are viewed as maps from $H \hat{\otimes} H$ to $M(H \hat{\otimes} H)$. In particular, the image of the last map is contained in $H \hat{\otimes} H$. We compute

$$
\begin{gathered}
\mu_{(2)}\left(\gamma_{r}^{-1} \gamma_{r} \hat{\otimes} \operatorname{id}_{(2)}\right)=\mu_{(2)}\left(\operatorname{id} \hat{\otimes} \mu \hat{\otimes} \operatorname{id}_{(2)}\right)\left(\operatorname{id} \hat{\otimes} S \hat{\otimes} \operatorname{id}_{(3)}\right)\left(\Delta \hat{\otimes} \operatorname{id}_{(3)}\right)\left(\gamma_{r} \hat{\otimes} \operatorname{id}_{(2)}\right) \\
=\mu_{(2)}\left(\operatorname{id} \hat{\otimes} \epsilon \hat{\otimes} \operatorname{id}_{(3)}\right)\left(\Delta \hat{\otimes} \operatorname{id}_{(3)}\right)=\mu_{(2)},
\end{gathered}
$$

where we write $\mu_{(2)}$ for the multiplication in the tensor product $H \hat{\otimes} H$. In a similar way one shows $\mu_{(2)}\left(\operatorname{id}_{(2)} \hat{\otimes} \gamma_{r} \gamma_{r}^{-1}\right)=\mu_{(2)}$ which shows that $\gamma_{r}$ is an isomorphism.

For the other Galois maps one could perform similar calculations. We proceed differently and show first that the given counit and invertible antipode for $H$ yield counits and antipodes for $H^{\mathrm{op}}, H^{\mathrm{cop}}$ and $H^{\mathrm{opcop}}$ as well. Observe that the counit $\epsilon$ satisfies

$$
(\epsilon \hat{\otimes} \mathrm{id}) \Delta^{\mathrm{cop}}=\mathrm{id}=(\mathrm{id} \hat{\otimes} \epsilon) \Delta^{\mathrm{cop}},
$$

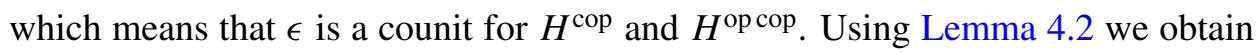

$$
\begin{aligned}
& \mu^{\mathrm{op}}(S \hat{\otimes} \mathrm{id}) \gamma_{r}^{\mathrm{op} \mathrm{cop}}=\mu \tau(S \hat{\otimes} \mathrm{id}) \tau \rho_{l} \tau=\mu(\mathrm{id} \hat{\otimes} S) \rho_{l} \tau=\epsilon \hat{\otimes} \mathrm{id}, \\
& \mu^{\mathrm{op}}(\mathrm{id} \hat{\otimes} S) \rho_{l}^{\mathrm{op} \mathrm{cop}}=\mu \tau(\mathrm{id} \hat{\otimes} S) \tau \gamma_{r} \tau=\mu(\mathrm{id} \hat{\otimes} S) \gamma_{r} \tau=\mathrm{id} \hat{\otimes} \epsilon,
\end{aligned}
$$

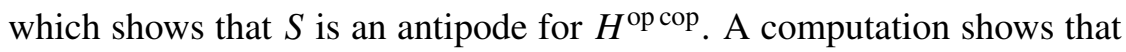

$$
\mu\left(\mu^{\mathrm{op}} \hat{\otimes} \mathrm{id}\right)\left(S^{-1} \hat{\otimes} \mathrm{id} \hat{\otimes} \mathrm{id}\right)\left(\gamma_{r}^{\mathrm{op}} \hat{\otimes} \mathrm{id}\right)=\mu(\epsilon \hat{\otimes} \mathrm{id} \hat{\otimes} \mathrm{id}),
$$


which yields

$$
\mu^{\mathrm{op}}\left(S^{-1} \hat{\otimes} \mathrm{id}\right) \gamma_{r}^{\mathrm{op}}=\epsilon \hat{\otimes} \mathrm{id} .
$$

Similarly we have $\mu\left(\mathrm{id} \hat{\otimes} \mu^{\mathrm{op}}\right)\left(\mathrm{id} \hat{\otimes} \operatorname{id} \hat{\otimes} S^{-1}\right)\left(\operatorname{id} \hat{\otimes} \rho_{l}^{\mathrm{op}}\right)=\mu(\mathrm{id} \hat{\otimes} \mathrm{id} \hat{\otimes} \epsilon)$, which implies

$$
\mu^{\mathrm{op}}\left(\mathrm{id} \hat{\otimes} S^{-1}\right) \rho_{l}^{\mathrm{op}}=\mathrm{id} \hat{\otimes} \epsilon .
$$

Hence $S^{-1}$ is an antipode for $H^{\text {op }}$. As above it follows that $S^{-1}$ is also an antipode for $H^{\text {cop }}=\left(H^{\text {op }}\right)^{\text {op cop }}$. We may now apply our previous argument for the Galois map $\gamma_{r}$ to $H^{\text {op }}, H^{\text {cop }}$ and $H^{\text {op cop }}$ and use Lemma 4.2 to see that $\gamma_{l}, \rho_{l}$ and $\rho_{r}$ are isomorphisms as well. This shows that $H$ is a bornological quantum group.

Conversely, let us assume that $H$ is a bornological quantum group and construct the maps $\epsilon$ and $S$. We begin with the counit $\epsilon$. Choose an element $h \in H$ such that $\phi(h)=1$ and set

$$
\epsilon(f)=\phi\left(\mu \rho_{l}^{-1}(h \otimes f)\right) .
$$

This obviously yields a bounded linear map $\epsilon: H \rightarrow \mathbb{C}$. Using

$$
\gamma_{r}(\operatorname{id} \hat{\otimes} \mu)=(\operatorname{id} \hat{\otimes} \mu) \gamma_{r},
$$

we easily see that the formula $E(f) \cdot g=\mu \gamma_{r}^{-1}(f \otimes g)$ defines a left multiplier $E(f)$ of $H$. Actually, we obtain a bounded linear map $E: H \rightarrow M_{l}(H)$ in this way. Using Lemma 4.5 we obtain

$(\mathrm{id} \hat{\otimes} \mu)(\mathrm{id} \hat{\otimes} E \hat{\otimes} \mathrm{id})\left(\rho_{l} \hat{\otimes} \mathrm{id}\right)\left(\mathrm{id} \hat{\otimes} \gamma_{r}\right)=(\mathrm{id} \hat{\otimes} \mu)\left(\mathrm{id} \hat{\otimes} \gamma_{r}^{-1}\right)\left(\rho_{l} \hat{\otimes} \mathrm{id}\right)\left(\mathrm{id} \hat{\otimes} \gamma_{r}\right)$

$$
\begin{aligned}
& =(\mathrm{id} \hat{\otimes} \mu)\left(\mathrm{id} \hat{\otimes} \gamma_{r}^{-1}\right)\left(\mathrm{id} \hat{\otimes} \gamma_{r}\right)\left(\rho_{l} \hat{\otimes} \mathrm{id}\right) \\
& =(\mu \hat{\otimes} \mathrm{id})\left(\mathrm{id} \hat{\otimes} \gamma_{r}\right) .
\end{aligned}
$$

Since $\gamma_{r}$ and $\rho_{l}$ are isomorphisms, this implies

$$
(\mathrm{id} \hat{\otimes} \mu)(\mathrm{id} \hat{\otimes} E \hat{\otimes} \mathrm{id})=\mu \rho_{l}^{-1} \hat{\otimes} \mathrm{id} .
$$

Evaluating (4-2) on a tensor $h \otimes f \otimes g$ where $h$ is chosen as above and applying $\phi \hat{\otimes}$ id we get

$$
E(f) \cdot g=(\phi \hat{\otimes} \mathrm{id})(h \otimes E(f) \cdot g)=\phi\left(\mu \rho_{l}^{-1}(h \otimes f)\right) g=\epsilon(f) g
$$

and hence

$$
E(f)=\epsilon(f) 1
$$

in $M_{l}(H)$ for every $f \in H$. Thus we could have used any nonzero bounded linear functional in order to define $\epsilon$.

According to (4-3) and the definition of $E$ we have

$$
(\epsilon \hat{\otimes} \mathrm{id}) \gamma_{r}=\mu(E \hat{\otimes} \mathrm{id}) \gamma_{r}=\mu .
$$


Equation (4-2) yields $g \epsilon(f) \otimes h=g \otimes \epsilon(f) h=\mu \rho_{l}^{-1}(g \otimes f) \otimes h$ for all $f, g \in H$, which implies

$$
g \epsilon(f)=\mu \rho_{l}^{-1}(g \otimes f) .
$$

This is equivalent to

$$
(\text { id } \hat{\otimes} \epsilon) \rho_{l}=\mu,
$$

since $\rho_{l}$ is an isomorphism.

We now show that $\epsilon$ is an algebra homomorphism. We have

$$
\rho_{l}(\mathrm{id} \hat{\otimes} \mu)=(\mu \hat{\otimes} \mathrm{id})\left(\mathrm{id} \hat{\otimes} \mu_{(2)}\right)(\mathrm{id} \hat{\otimes} \Delta \hat{\otimes} \Delta)=\mu_{(2)}\left(\rho_{l} \hat{\otimes} \Delta\right),
$$

because $\Delta$ is an algebra homomorphism. From this relation and (4-6) we get

$(\mathrm{id} \hat{\otimes} \epsilon) \mu_{(2)}(\mathrm{id} \hat{\otimes} \mathrm{id} \hat{\otimes} \Delta)\left(\rho_{l} \hat{\otimes} \mathrm{id}\right)=(\mathrm{id} \hat{\otimes} \epsilon) \rho_{l}(\mathrm{id} \hat{\otimes} \mu)=\mu(\mathrm{id} \hat{\otimes} \mu)$

$$
=\mu(\mu \hat{\otimes} \mathrm{id})=\mu(\mathrm{id} \hat{\otimes} \epsilon \hat{\otimes} \mathrm{id})\left(\rho_{l} \hat{\otimes} \mathrm{id}\right),
$$

and since $\rho_{l}$ is an isomorphism this implies

$$
(\mathrm{id} \hat{\otimes} \epsilon) \mu_{(2)}(\mathrm{id} \hat{\otimes} \mathrm{id} \hat{\otimes} \Delta)=\mu(\mathrm{id} \hat{\otimes} \epsilon \hat{\otimes} \mathrm{id}) .
$$

Now observe $\mu_{(2)}(\mathrm{id} \hat{\otimes} \mathrm{id} \hat{\otimes} \Delta)=(\operatorname{id} \hat{\otimes} \mu) \rho_{l}^{13}$ and hence

$$
(\operatorname{id} \hat{\otimes} \epsilon)(\operatorname{id} \hat{\otimes} \mu) \rho_{l}^{13}=\mu(\operatorname{id} \hat{\otimes} \epsilon \hat{\otimes} \text { id })=(\operatorname{id} \hat{\otimes} \epsilon)(\operatorname{id} \hat{\otimes} \epsilon \hat{\otimes} \text { id }) \rho_{l}^{13},
$$

where we use (4-6). We deduce (id $\hat{\otimes} \epsilon)(\mathrm{id} \hat{\otimes} \mu)=(\mathrm{id} \hat{\otimes} \epsilon)(\mathrm{id} \hat{\otimes} \epsilon \hat{\otimes} \mathrm{id})$, which implies $\epsilon(f g)=\epsilon(f) \epsilon(g)$ for all $f, g \in H$. Thus $\epsilon$ is an algebra homomorphism.

Using this fact and (4-6) we calculate

$\mu\left(\operatorname{id}_{(2)} \hat{\otimes} \epsilon\right)\left(\mathrm{id} \hat{\otimes} \gamma_{r}\right)=(\mathrm{id} \hat{\otimes} \epsilon)(\operatorname{id} \hat{\otimes} \mu)\left(\rho_{l} \hat{\otimes} \mathrm{id}\right)=(\mathrm{id} \hat{\otimes} \mu)(\operatorname{id} \hat{\otimes} \epsilon \hat{\otimes} \epsilon)\left(\rho_{l} \hat{\otimes} \mathrm{id}\right)=\mu \hat{\otimes} \epsilon$, which implies

$$
(\operatorname{id} \hat{\otimes} \epsilon) \gamma_{r}=\operatorname{id} \hat{\otimes} \epsilon \text {. }
$$

Analogously,

$$
(\epsilon \hat{\otimes} \mathrm{id}) \rho_{l}=\epsilon \hat{\otimes} \mathrm{id}
$$

as a consequence of (4-4).

It is easy to see that the map $\epsilon$ is nonzero. To check that $\epsilon$ is nondegenerate we define a bounded linear map $\sigma: \mathbb{C} \rightarrow H \hat{\otimes}_{H} \mathbb{C}$ by $\sigma(1)=k \otimes 1$ where $k \in H$ is an element satisfying $\epsilon(k)=1$. Using (4-4) and (4-8) we obtain

$$
\begin{aligned}
\sigma(\epsilon \otimes \mathrm{id})(f \otimes 1) & =\epsilon(f) k \otimes 1=\mu \gamma_{r}^{-1}(f \otimes k) \otimes 1 \\
& =(\operatorname{id} \hat{\otimes} \epsilon) \gamma_{r}^{-1}(f \otimes k)=f \otimes \epsilon(k) 1=f \otimes 1,
\end{aligned}
$$


which implies $H \hat{\otimes}_{H} \mathbb{C} \cong \mathbb{C}$. In a similar way one checks $\mathbb{C} \hat{\otimes}_{H} H \cong \mathbb{C}$ using (4-9). This shows that $\epsilon$ is nondegenerate.

According to (4-6) we thus have

$$
(\mathrm{id} \hat{\otimes} \epsilon) \Delta=\mathrm{id},
$$

and using (4-4) we get

$$
(\epsilon \hat{\otimes} \mathrm{id}) \Delta=\mathrm{id} .
$$

Conversely, the last equation implies $(\epsilon \hat{\otimes}$ id $) \gamma_{r}=\mu$ which in turn determines $\epsilon$ uniquely since $\gamma_{r}$ is an isomorphism.

Now we shall construct the antipode. It is easy to check that the formulas

$$
S_{l}(f) \cdot g=(\epsilon \hat{\otimes} \mathrm{id}) \gamma_{r}^{-1}(f \otimes g), \quad g \cdot S_{r}(f)=(\mathrm{id} \hat{\otimes} \epsilon) \rho_{l}^{-1}(g \otimes f)
$$

define a left multiplier $S_{l}(f)$ and a right multiplier $S_{r}(f)$ of $H$ for every $f \in H$. In this way we obtain bounded linear maps $S_{l}: H \rightarrow M_{l}(H)$ and $S_{r}: H \rightarrow M_{r}(H)$.

Let us show that $S_{l}$ is an algebra antihomomorphism. Using Lemma 4.5 and (4-6) we calculate

$$
(\mathrm{id} \hat{\otimes} \mu)\left(\mathrm{id} \hat{\otimes} S_{l} \hat{\otimes} \mathrm{id}\right)\left(\rho_{l} \hat{\otimes} \mathrm{id}\right)=(\mu \hat{\otimes} \mathrm{id})\left(\mathrm{id} \hat{\otimes} \gamma_{r}^{-1}\right) .
$$

Applying the multiplication map $\mu$ to this equation yields

$$
\mu(\mathrm{id} \hat{\otimes} \mu)\left(\mathrm{id} \hat{\otimes} S_{l} \hat{\otimes} \mathrm{id}\right)\left(\rho_{l} \hat{\otimes} \mathrm{id}\right)=\mu\left(\mathrm{id} \hat{\otimes} \mu \gamma_{r}^{-1}\right)=\mu(\mathrm{id} \hat{\otimes} \epsilon \hat{\otimes} \mathrm{id}),
$$

where we have used (4-4). Applying (4-7), (4-12), the fact that $\epsilon$ is an algebra homomorphism, and again (4-12), we obtain

$$
\begin{aligned}
& \mu(\mathrm{id} \hat{\otimes} \mu)\left(\mathrm{id} \hat{\otimes} S_{l} \hat{\otimes} \mathrm{id}\right)\left(\mu_{(2)} \hat{\otimes} \mathrm{id}\right)\left(\rho_{l} \hat{\otimes} \Delta \hat{\otimes} \mathrm{id}\right) \\
& \quad=\mu(\mathrm{id} \hat{\otimes} \mu)\left(\mathrm{id} \hat{\otimes} S_{l} \hat{\otimes} \mathrm{id}\right)\left(\rho_{l} \hat{\otimes} \mathrm{id}\right)(\mathrm{id} \hat{\otimes} \mathrm{id} \hat{\otimes} \epsilon \hat{\otimes} \mathrm{id}) .
\end{aligned}
$$

Since $\rho_{l}$ is an isomorphism this yields, due to (4-12),

$\mu(\mathrm{id} \hat{\otimes} \mu)\left(\mathrm{id} \hat{\otimes} S_{l} \hat{\otimes} \mathrm{id}\right)(\mathrm{id} \hat{\otimes} \mu \hat{\otimes} \mathrm{id}) \rho_{l}^{13}$

$$
=\mu(\mathrm{id} \hat{\otimes} \mu)\left(\mathrm{id} \hat{\otimes} S_{l} \hat{\otimes} \mathrm{id}\right)\left(\mathrm{id}_{(2)} \hat{\otimes} \mu\right)\left(\mathrm{id}_{(2)} \hat{\otimes} S_{l} \hat{\otimes} \mathrm{id}\right)(\mathrm{id} \hat{\otimes} \tau \hat{\otimes} \mathrm{id}) \rho_{l}^{13},
$$

and hence

$\mu(\mathrm{id} \hat{\otimes} \mu)\left(\mathrm{id} \hat{\otimes} S_{l} \hat{\otimes} \mathrm{id}\right)(\mathrm{id} \hat{\otimes} \mu \hat{\otimes} \mathrm{id})$

$$
=\mu(\mathrm{id} \hat{\otimes} \mu)(\mathrm{id} \hat{\otimes} \mu \hat{\otimes} \mathrm{id})(\mathrm{id} \hat{\otimes} \tau \hat{\otimes} \mathrm{id})\left(\mathrm{id} \hat{\otimes} S_{l} \hat{\otimes} S_{l} \hat{\otimes} \mathrm{id}\right) .
$$

Since the algebra $H$ is nondegenerate we obtain

$$
S_{l}(f g)=S_{l}(g) S_{l}(f)
$$

for all $f, g \in H$, as claimed. 
For the map $S_{r}$ we do an analogous calculation. We have $(\mu \hat{\otimes} \mathrm{id})\left(\mathrm{id} \hat{\otimes} S_{r} \hat{\otimes} \mathrm{id}\right)\left(\mathrm{id} \hat{\otimes} \gamma_{r}\right)=(\mu \hat{\otimes} \mathrm{id})\left(\mathrm{id} \hat{\otimes} S_{r} \hat{\otimes} \mathrm{id}\right)\left(\rho_{l} \hat{\otimes} \mathrm{id}\right)\left(\mathrm{id} \hat{\otimes} \gamma_{r}\right)\left(\rho_{l}^{-1} \hat{\otimes} \mathrm{id}\right)$

$$
\begin{aligned}
& =(\mathrm{id} \hat{\otimes} \epsilon \hat{\otimes} \mathrm{id})\left(\mathrm{id} \hat{\otimes} \gamma_{r}\right)\left(\rho_{l}^{-1} \hat{\otimes} \mathrm{id}\right) \\
& =(\mathrm{id} \hat{\otimes} \mu)\left(\rho_{l}^{-1} \hat{\otimes} \mathrm{id}\right),
\end{aligned}
$$

and applying $\mu$ yields

$$
\mu(\mu \hat{\otimes} \mathrm{id})\left(\mathrm{id} \hat{\otimes} S_{r} \hat{\otimes} \mathrm{id}\right)\left(\mathrm{id} \hat{\otimes} \gamma_{r}\right)=\mu\left(\mu \rho_{l}^{-1} \hat{\otimes} \mathrm{id}\right)=\mu(\mathrm{id} \hat{\otimes} \epsilon \hat{\otimes} \mathrm{id}) .
$$

As above one may proceed to show that $S_{r}$ is an algebra antihomomorphism. We shall instead first show that $\left(S_{l}(f), S_{r}(f)\right)$ is a two-sided multiplier of $H$ for every $f \in H$. By the definition of $S_{r}$ we have $\mu\left(\operatorname{id} \hat{\otimes} S_{r}\right)=(\operatorname{id} \hat{\otimes} \epsilon) \rho_{l}^{-1}$ and hence (4-12) implies

$$
\mu(\mathrm{id} \hat{\otimes} \mu)\left(\mathrm{id} \hat{\otimes} S_{l} \hat{\otimes} \mathrm{id}\right)=\mu(\mathrm{id} \hat{\otimes} \epsilon \hat{\otimes} \mathrm{id})\left(\rho_{l}^{-1} \hat{\otimes} \mathrm{id}\right)=\mu(\mu \hat{\otimes} \mathrm{id})\left(\mathrm{id} \hat{\otimes} S_{r} \hat{\otimes} \mathrm{id}\right),
$$

which is precisely the required identity. We can now use (4-13) to obtain that $S_{r}$ is an algebra antihomomorphism. If $S: H \rightarrow M(H)$ denotes the linear map determined by $S_{l}$ and $S_{r}$ we have thus showed so far that $S: H \rightarrow M(H)$ is a bounded algebra antihomomorphism.

For $f \in H$, define $\bar{S}_{l}(f) \in M_{l}(H)$ and $\bar{S}_{r}(f) \in M_{r}(H)$ by

$$
\begin{aligned}
& \bar{S}_{l}(f) \cdot g=(\epsilon \hat{\otimes} \mathrm{id}) \gamma_{l}^{-1} \tau(f \otimes g), \\
& g \cdot \bar{S}_{r}(f)=(\operatorname{id} \hat{\otimes} \epsilon) \rho_{r}^{-1} \tau(g \otimes f) .
\end{aligned}
$$

According to Lemma 4.2 we have $\gamma_{l}^{-1} \tau=\left(\gamma_{r}^{\text {cop }}\right)^{-1}$ and $\rho_{r}^{-1} \tau=\left(\rho_{l}^{\text {cop }}\right)^{-1}$. The discussion above applied to $H^{\text {cop }}$ shows that $\bar{S}_{l}$ and $\bar{S}_{r}$ determine a bounded algebra antihomomorphism $\bar{S}: H \rightarrow M(H)$.

Our next goal is to prove that $S$ and $\bar{S}$ actually define bounded linear maps from $H$ into itself which are inverse to each other. To do this, observe that

$$
\begin{aligned}
(\mathrm{id} \hat{\otimes} \mu)(\tau \hat{\otimes} \mathrm{id}) & =(\mathrm{id} \hat{\otimes} \mu)(\tau \hat{\otimes} \mathrm{id})\left(\gamma_{r} \hat{\otimes} \mathrm{id}\right)\left(\gamma_{r}^{-1} \hat{\otimes} \mathrm{id}\right) \\
& =\mu_{(2)}\left(\Delta^{\mathrm{cop}} \hat{\otimes} \mathrm{id}_{(2)}\right)\left(\gamma_{r}^{-1} \hat{\otimes} \mathrm{id}\right),
\end{aligned}
$$

which implies

$(\mu \hat{\otimes} \mathrm{id})(\mathrm{id} \hat{\otimes} \bar{S} \hat{\otimes} \mathrm{id})(\mathrm{id} \hat{\otimes} \mathrm{id} \hat{\otimes} \mu)(\mathrm{id} \hat{\otimes} \tau \hat{\otimes} \mathrm{id})$

$$
=(\mu \hat{\otimes} \mathrm{id})(\mathrm{id} \hat{\otimes} \mu \hat{\otimes} \mathrm{id})(\mathrm{id} \hat{\otimes} \bar{S} \hat{\otimes} \bar{S} \hat{\otimes} \mathrm{id})\left(\mathrm{id}_{(2)} \hat{\otimes} \gamma_{r}^{\mathrm{cop}} \hat{\otimes} \mathrm{id}\right)\left(\mathrm{id} \hat{\otimes} \tau \gamma_{r}^{-1} \hat{\otimes} \mathrm{id}\right),
$$

since $\bar{S}$ is an algebra antihomomorphism. Applying $\mu$ to this equation and inserting the definitions of $S$ and $\bar{S}$ we calculate

$\mu(\mathrm{id} \hat{\otimes} \mu)(\mathrm{id} \hat{\otimes} \mu \hat{\otimes} \mathrm{id})\left(\mathrm{id} \hat{\otimes} \bar{S} \hat{\otimes} \mathrm{id}_{(2)}\right)(\mathrm{id} \hat{\otimes} \tau \hat{\otimes} \mathrm{id})$

$$
=\mu(\mathrm{id} \hat{\otimes} \mu)(\mathrm{id} \hat{\otimes} \bar{S} \hat{\otimes} \mathrm{id})(\mathrm{id} \hat{\otimes} \mu \hat{\otimes} \mathrm{id})\left(\mathrm{id} \hat{\otimes} S \hat{\otimes} \mathrm{id}_{(2)}\right) \text {. }
$$


As a consequence we obtain the relation

$$
\mu(\bar{S} \hat{\otimes} \mathrm{id}) \tau=\bar{S} \mu(S \hat{\otimes} \mathrm{id}),
$$

where both sides are viewed as maps from $H \hat{\otimes} H$ into $M(H)$. Choose $k \in H$ such that $\epsilon(k)=1$. Equation (4-15) together with the definition of $S$ yields the relation

$$
\bar{S}(f)=\bar{S}(f) \epsilon(k)=\bar{S} \mu(S \hat{\otimes} \mathrm{id}) \gamma_{r}(k \otimes f)=\mu(\bar{S} \hat{\otimes} \mathrm{id}) \tau \gamma_{r}(k \otimes f)
$$

for all $f \in H$. This shows that $\bar{S}$ defines a bounded linear map from $H$ to $H$. Replacing $H$ by $H^{\text {cop }}$ we see that $S$ may be viewed as a bounded linear map from $H$ to $H$ as well. Since $\bar{S}$ is an algebra antihomomorphism, (4-15) then yields $\mu(\bar{S} \hat{\otimes} \mathrm{id})=\mu(\bar{S} \hat{\otimes} \bar{S} S)$ and hence

$$
\mu(\mu \hat{\otimes} \mathrm{id})(\mathrm{id} \hat{\otimes} \bar{S} \hat{\otimes} \mathrm{id})=\mu(\mu \hat{\otimes} \mathrm{id})(\mathrm{id} \hat{\otimes} \bar{S} \hat{\otimes} \bar{S} S) .
$$

Since $\mu(\operatorname{id} \hat{\otimes} \bar{S})=(\operatorname{id} \hat{\otimes} \epsilon) \rho_{r}^{-1} \tau$ by the definition of $\bar{S}$ we get $\bar{S} S=$ id. Analogously one obtains $S \bar{S}=$ id. Equations (4-14) and (4-12) yield

$$
\mu(S \hat{\otimes} \mathrm{id}) \gamma_{r}=\epsilon \hat{\otimes} \mathrm{id}, \quad \mu(\mathrm{id} \hat{\otimes} S) \rho_{l}=\mathrm{id} \hat{\otimes} \epsilon,
$$

as desired. Moreover these equations determine the map $S$ uniquely.

We show that $S$ is a coalgebra antihomomorphism. Since $\Delta$ is an algebra homomorphism we have

$$
\gamma_{r}(\mu \hat{\otimes} \mathrm{id})=(\mathrm{id} \hat{\otimes} \mu)(\Delta \hat{\otimes} \mathrm{id})(\mu \hat{\otimes} \mathrm{id})=(\mathrm{id} \hat{\otimes} \mu)\left(\gamma_{l} \hat{\otimes} \mathrm{id}\right)\left(\mathrm{id} \hat{\otimes} \gamma_{r}\right)
$$

and using (4-1) we get

$$
(\mu \hat{\otimes} \mathrm{id})\left(\mathrm{id} \hat{\otimes} \gamma_{r}^{-1}\right)=(\mathrm{id} \hat{\otimes} \mu)\left(\gamma_{r}^{-1} \hat{\otimes} \mathrm{id}\right)\left(\gamma_{l} \hat{\otimes} \mathrm{id}\right) .
$$

According to Lemma 4.5, (4-6) and the definition of $S$ we get

$$
(\mu \hat{\otimes} \mathrm{id})\left(\mathrm{id} \hat{\otimes} \gamma_{r}^{-1}\right)=(\mathrm{id} \hat{\otimes} \mu)(\mathrm{id} \hat{\otimes} S \hat{\otimes} \mathrm{id})\left(\rho_{l} \hat{\otimes} \mathrm{id}\right) .
$$

Together with (4-16) we obtain

$$
\gamma_{r}^{-1} \gamma_{l}=(\operatorname{id} \hat{\otimes} S) \rho_{l},
$$

which, applied to $H^{\text {cop }}$, yields

$$
\gamma_{l}^{-1} \gamma_{r}=\left(\gamma_{r}^{\mathrm{cop}}\right)^{-1} \tau \tau \gamma_{l}^{\mathrm{cop}}=\left(\mathrm{id} \hat{\otimes} S^{-1}\right) \rho_{l}^{\mathrm{cop}}=\left(\mathrm{id} \hat{\otimes} S^{-1}\right) \tau \rho_{r} .
$$

Equations (4-17) and (4-18) imply

$$
\rho_{r}(S \hat{\otimes} S)=(S \hat{\otimes} \mathrm{id}) \tau \rho_{l}^{-1}(S \hat{\otimes} \mathrm{id}) .
$$

From the definition of $S$ and (4-4) we have $(\mu \hat{\otimes} \mathrm{id})(\mathrm{id} \hat{\otimes} S \hat{\otimes} \mathrm{id})\left(\mathrm{id} \hat{\otimes} \gamma_{r}\right)\left(\rho_{l} \hat{\otimes} \mathrm{id}\right)=(\mathrm{id} \hat{\otimes} \epsilon \hat{\otimes} \mathrm{id})\left(\mathrm{id} \hat{\otimes} \gamma_{r}\right)=(\mathrm{id} \hat{\otimes} \mu)$. 
This implies

$$
(\mathrm{id} \hat{\otimes} \mu)\left(\rho_{l}^{-1} \hat{\otimes} \mathrm{id}\right)(\tau \hat{\otimes} \mathrm{id})(\mathrm{id} \hat{\otimes} S \hat{\otimes} \mathrm{id})=(\mathrm{id} \hat{\otimes} \mu)\left(S \hat{\otimes} \mathrm{id}_{(2)}\right)\left(\gamma_{l} \hat{\otimes} \mathrm{id}\right),
$$

since $S$ is an algebra antihomomorphism. Consequently we obtain

$$
(S \hat{\otimes} \mathrm{id}) \gamma_{l}=\rho_{l}^{-1}(S \hat{\otimes} \mathrm{id}) \tau .
$$

Equations (4-19) and (4-20) yield

$$
\rho_{r}(S \hat{\otimes} S) \tau=(S \hat{\otimes} \mathrm{id}) \tau \rho_{l}^{-1}(S \hat{\otimes} \mathrm{id}) \tau=(S \hat{\otimes} S) \tau \gamma_{l},
$$

and using that $S$ is an algebra antihomomorphism another computation gives $\left(\mathrm{id} \hat{\otimes} \mu^{\mathrm{op}}\right)(\Delta \hat{\otimes} \mathrm{id})(S \hat{\otimes} \mathrm{id})(\mathrm{id} \hat{\otimes} S)$

$$
=\left(\mathrm{id} \hat{\otimes} \mu^{\mathrm{op}}\right)(S \hat{\otimes} S \hat{\otimes} \mathrm{id})(\tau \hat{\otimes} \mathrm{id})(\Delta \hat{\otimes} \mathrm{id})(\mathrm{id} \hat{\otimes} S) .
$$

This implies $\Delta S=(S \hat{\otimes} S) \tau \Delta$ and shows that $S$ is a coalgebra antihomomorphism. Of course $S^{-1}$ is a coalgebra antihomomorphism as well. Thus we have shown that there exist unique maps $S$ and $\epsilon$ with the desired properties.

Recall that a morphism of bornological quantum groups is an essential algebra homomorphism $\alpha: H \rightarrow M(K)$ which is also a coalgebra homomorphism.

Proposition 4.7. Every morphism $\alpha: H \rightarrow M(K)$ of bornological quantum groups is automatically compatible with the counits and the antipodes.

Proof. Note that the Galois maps associated to the comultiplication of $H$ extend to bounded linear maps from $M(H) \hat{\otimes} M(H)$ into $M(H \hat{\otimes} H)$. Moreover observe that the relation $(\epsilon \hat{\otimes}$ id $) \gamma_{r}=\mu$ obtained in (4-4) still holds when we consider both sides as maps form $M(H) \hat{\otimes} M(H)$ to $M(H)$.

Since $\alpha: H \rightarrow M(K)$ is an algebra homomorphism and a coalgebra homomorphism we have

$$
\gamma_{r}(\alpha \hat{\otimes} \alpha)=(\alpha \hat{\otimes} \alpha) \gamma_{r},
$$

where both sides are viewed as maps from $H \hat{\otimes} H$ into $M(K \hat{\otimes} K)$. Hence we obtain

$$
(\epsilon \hat{\otimes} \mathrm{id})(\alpha \hat{\otimes} \alpha) \gamma_{r}=(\epsilon \hat{\otimes} \mathrm{id}) \gamma_{r}(\alpha \hat{\otimes} \alpha)=\mu(\alpha \hat{\otimes} \alpha)=\alpha \mu=(\epsilon \hat{\otimes} \alpha) \gamma_{r},
$$

where all maps are considered to be defined on $H \hat{\otimes} H$ with values in $M(K)$. We conclude $(\epsilon \alpha) \hat{\otimes} \alpha=\epsilon \hat{\otimes} \alpha$ because $\gamma_{r}$ is an isomorphism. Since $\alpha$ is nondegenerate this shows $\epsilon \alpha=\epsilon$ which means that $\alpha$ is compatible with the counits.

The arguments given in the proof of Theorem 4.6 show that the inverses of the Galois maps of $H$ can be described explicitly using the antipode $S$ and its inverse. It follows that these maps are defined on $M(H) \hat{\otimes} M(H)$ in a natural way. 
With this in mind and using the equality $(\mu \hat{\otimes} \mathrm{id})\left(\mathrm{id} \hat{\otimes} \rho_{r}\right)(\mathrm{id} \hat{\otimes} \mu \hat{\otimes} \mathrm{id})(\mathrm{id} \hat{\otimes} \tau \hat{\otimes} \mathrm{id})\left(\rho_{l} \hat{\otimes} \mathrm{id}_{(2)}\right)$

$$
=\mu_{(2)}\left(\operatorname{id}_{(2)} \hat{\otimes} \mu_{(2)}\right)\left(\operatorname{id}_{(2)} \hat{\otimes} \Delta \hat{\otimes} \Delta\right)(\mathrm{id} \hat{\otimes} \tau \hat{\otimes} \mathrm{id}),
$$

we compute on $H \hat{\otimes} H \hat{\otimes} M(H) \hat{\otimes} H$ the relation

$(\mu \hat{\otimes} \mathrm{id})(\mathrm{id} \hat{\otimes} \mu \hat{\otimes} \mathrm{id})\left(\mathrm{id}_{(2)} \hat{\otimes} \gamma_{r}^{-1}\right)=(\mu \hat{\otimes} \mathrm{id})\left(\mathrm{id} \hat{\otimes} \gamma_{r}^{-1}\right)(\mathrm{id} \hat{\otimes} \mu \hat{\otimes} \mathrm{id}) \gamma_{r}^{24}$.

This yields $(\mu \hat{\otimes} \mathrm{id})\left(\mathrm{id} \hat{\otimes} \gamma_{r}^{-1}\right)=\gamma_{r}^{-1}(\mu \hat{\otimes} \mathrm{id}) \gamma_{r}^{13}$ on $H \hat{\otimes} M(H) \hat{\otimes} H$, which in turn implies

$$
(\mu \hat{\otimes} \mu)\left(\mathrm{id} \hat{\otimes} \gamma_{r}^{-1} \hat{\otimes} \mathrm{id}\right)=\gamma_{r}^{-1}(\mu \hat{\otimes} \mathrm{id}) \gamma_{r}^{13}\left(\mathrm{id}_{(2)} \hat{\otimes} \mu\right)
$$

if both sides are viewed as maps from $H \hat{\otimes} M(H) \hat{\otimes} M(H) \hat{\otimes} H$ into $H \hat{\otimes} H$. Moreover,

$\gamma_{r}(\mu \hat{\otimes} \mu)(\mathrm{id} \hat{\otimes} \alpha \hat{\otimes} \alpha \hat{\otimes} \mathrm{id})$

$$
\begin{aligned}
& =(\mathrm{id} \hat{\otimes} \mu)\left(\operatorname{id}_{(2)} \hat{\otimes} \mu\right)\left(\Delta \hat{\otimes} \mathrm{id}_{(2)}\right)\left(\mu \hat{\otimes} \mathrm{id}_{(2)}\right)(\mathrm{id} \hat{\otimes} \alpha \hat{\otimes} \alpha \hat{\otimes} \mathrm{id}) \\
& =(\mu \hat{\otimes} \mathrm{id}) \gamma_{r}^{13}\left(\mathrm{id}_{(2)} \hat{\otimes} \mu\right)(\mathrm{id} \hat{\otimes} \alpha \hat{\otimes} \alpha \hat{\otimes} \mathrm{id})\left(\mathrm{id} \hat{\otimes} \gamma_{r} \hat{\otimes} \mathrm{id}\right)
\end{aligned}
$$

as maps from $K \hat{\otimes} H \hat{\otimes} H \hat{\otimes} K$ into $K \hat{\otimes} K$, which yields, according to (4-22), $(\mu \hat{\otimes} \mu)(\mathrm{id} \hat{\otimes} \alpha \hat{\otimes} \alpha \hat{\otimes} \mathrm{id})=\gamma_{r}^{-1} \gamma_{r}(\mu \hat{\otimes} \mu)(\mathrm{id} \hat{\otimes} \alpha \hat{\otimes} \alpha \hat{\otimes} \mathrm{id})$

$$
=(\mu \hat{\otimes} \mu)\left(\mathrm{id} \hat{\otimes} \gamma_{r}^{-1} \hat{\otimes} \mathrm{id}\right)(\mathrm{id} \hat{\otimes} \alpha \hat{\otimes} \alpha \hat{\otimes} \mathrm{id})\left(\mathrm{id} \hat{\otimes} \gamma_{r} \hat{\otimes} \mathrm{id}\right)
$$

and we deduce

$$
\gamma_{r}^{-1}(\alpha \hat{\otimes} \alpha)=(\alpha \hat{\otimes} \alpha) \gamma_{r}^{-1} .
$$

Note that this assertion does not immediately follow from (4-21) since the map $\gamma_{r}^{-1}$ is not defined on the multiplier algebra $M(K \hat{\otimes} K)$.

Now, the relation $\mu(S \hat{\otimes}$ id $)=(\epsilon \hat{\otimes}$ id $) \gamma_{r}^{-1}$ still holds if both sides are viewed as maps from $M(H) \hat{\otimes} M(H)$ into $M(H)$. Using this observation we obtain the relations

$$
\begin{aligned}
\mu(S \hat{\otimes} \mathrm{id})(\alpha \hat{\otimes} \alpha) & =(\epsilon \hat{\otimes} \mathrm{id}) \gamma_{r}^{-1}(\alpha \hat{\otimes} \alpha), \\
\alpha \mu(S \hat{\otimes} \mathrm{id}) & =(\epsilon \hat{\otimes} \alpha) \gamma_{r}^{-1},
\end{aligned}
$$

which, together with (4-23) and the fact that $\alpha$ is compatible with the counits, yield $\mu(\alpha S \hat{\otimes} \alpha)=\alpha \mu(S \hat{\otimes} \mathrm{id})=(\epsilon \hat{\otimes} \mathrm{id})(\alpha \hat{\otimes} \alpha) \gamma_{r}^{-1}=(\epsilon \hat{\otimes} \mathrm{id}) \gamma_{r}^{-1}(\alpha \hat{\otimes} \alpha)=\mu(S \alpha \hat{\otimes} \alpha)$.

Since $\alpha$ is nondegenerate it follows that the maps $\alpha S$ and $S \alpha$ coincide. Remark that we also have $\alpha S^{-1}=S^{-1} \alpha$. 
Proposition 4.7 implies in particular $\epsilon S=\epsilon$ and $\epsilon S^{-1}=\epsilon$ in the bornological quantum group $H$ since $S: H \rightarrow H^{\text {op cop }}$ is an isomorphism of bornological quantum groups.

\section{Modular properties of the integral}

In this section we discuss modular properties of the Haar functional on a bornological quantum group. Since the results and arguments are parallel to the ones for algebraic quantum groups [van Daele 1994; 1998] we omit the proofs.

Let $H$ be a bornological quantum group. If $\phi$ is a left invariant functional then $S(\phi)$ is right invariant where $S(\phi)(f)=\phi(S(f))$. In particular, there always exists a faithful right invariant functional on $H$.

Proposition 5.1. Let $H$ be a bornological quantum group and let $\phi$ and $\psi$ be faithful left and right invariant functionals on $H$, respectively. There exists a bornological isomorphism $v$ of $H$ such that

$$
\psi(h f)=\phi(h v(f))
$$

for all $f, h \in H$.

Proposition 5.2. The left Haar functional $\phi$ for a bornological quantum group $H$ is unique up to a scalar.

Proposition 5.3. Let $H$ be a bornological quantum group. There exists a unique bounded algebra automorphism $\sigma$ of $H$ such that

$$
\phi(f g)=\phi(g \sigma(f))
$$

for all $f, g \in H$. Moreover $\phi$ is invariant under $\sigma$.

Let $\psi$ be a right Haar measure on $H$. Then $S(\psi)$ is a left Haar measure and we obtain

$$
\left.\psi(S(f) S(g))=S(\psi)(g f)=S(\psi)\left(\sigma^{-1}(f) g\right)=\psi\left(S(g)\left(S \sigma^{-1} S^{-1}\right) S(f)\right)\right)
$$

for all $f, g \in H$, and thus

$$
\psi(f g)=\psi(g \rho(f)),
$$

where $\rho=S \sigma^{-1} S^{-1}$. If we also consider $S^{-1}(\psi)$ and use Proposition 5.2 we get $\rho=S^{-1} \sigma^{-1} S$ which yields the relation $S^{2} \sigma=\sigma S^{2}$ for the automorphism $\sigma$.

Proposition 5.4. Let $H$ be a bornological quantum group. There exists a unique multiplier $\delta \in M(H)$ such that

$$
(\phi \hat{\otimes} \mathrm{id}) \Delta(f)=\phi(f) \delta
$$

for all $f \in H$. 
The multiplier $\delta$ is called the modular element of $H$.

Proposition 5.5. The modular element $\delta$ is invertible and satisfies the relations

$$
\Delta(\delta)=\delta \otimes \delta, \quad \epsilon(\delta)=1, \quad S(\delta)=\delta^{-1} .
$$

Observe that we also have $S^{-1}(\delta)=\delta^{-1}$. If $\psi$ is a faithful right invariant functional then the formula

$$
(\mathrm{id} \hat{\otimes} \psi) \Delta(f)=\psi(f) \delta^{-1}
$$

describes the corresponding modular relation. This follows from Propositions 5.4 and 5.5 using the left invariant functional $\phi=S(\psi)$.

\section{Modules and comodules}

In this section we discuss the concepts of an essential module and an essential comodule over a bornological quantum group.

We begin with the notion of an essential module. Actually, the definition of essential modules over bornological algebras was already given in Section 3.

Definition 6.1. Let $H$ be a bornological quantum group. An essential $H$-module is an $H$-module $V$ such that the module action induces a bornological isomorphism $H \hat{\otimes}_{H} V \cong V$. A bounded linear map $f: V \rightarrow W$ between essential $H$-modules is called $H$-linear if $\mu_{W}($ id $\hat{\otimes} f)=f \mu_{V}$.

If $H$ and the $H$-module $V$ carry the fine bornology, then $V$ is essential if and only if $H V=V$. This follows easily from the fact that $H$ has an approximate identity in this case. Modules satisfying the condition $H V=V$ are called unital in [Drabant et al. 1999]. Hence unital modules over an algebraic quantum group are essential.

We denote the category of essential $H$-modules by $H$-Mod. By definition, the morphisms in $H$-Mod are the bounded $H$-linear maps.

The direct sum of a family of essential $H$-modules is again an essential $\mathrm{H}$ module. Moreover, the tensor product $V \hat{\otimes} W$ of two essential $H$-modules becomes an essential $H$-module using the diagonal action. The trivial one-dimensional $\mathrm{H}$ module $\mathbb{C}$ given by the counit $\epsilon$ behaves like a unit with respect to the tensor product.

Dually to the concept of an essential module one has the notion of an essential comodule. Let $H$ be a bornological quantum group, let $V$ be a bornological vector space and let $\operatorname{Hom}_{H}(H, V \hat{\otimes} H)$ be the space of bounded right $H$-linear maps from $H$ to $V \hat{\otimes} H$. A coaction of $H$ on $V$ is a bounded linear map $\eta: V \rightarrow \operatorname{Hom}_{H}(H, V \hat{\otimes}$ $H$ ) which is colinear in the following sense. By adjoint associativity, the map $\eta$ can equivalently be described as a bounded $H$-linear map $V \hat{\otimes} H \rightarrow V \hat{\otimes} H$. Then $\eta$ is 
said to be $H$-colinear if the latter map is an isomorphism and satisfies the relation

$$
\left(\mathrm{id} \otimes \gamma_{r}\right) \eta_{12}\left(\mathrm{id} \otimes \gamma_{r}^{-1}\right)=\eta_{12} \eta_{13}
$$

where both sides are viewed as maps from $V \hat{\otimes} H \hat{\otimes} H$ to itself.

Definition 6.2. Let $H$ be a bornological quantum group. An essential $H$-comodule is a bornological vector space $V$ together with a coaction $\eta: V \rightarrow \operatorname{Hom}_{H}(H, V \hat{\otimes}$ $H$ ). A bounded linear map $f: V \rightarrow W$ between essential comodules is called $H$-colinear if $(f \otimes \mathrm{id}) \eta_{V}=\eta_{W}(f \otimes \mathrm{id})$.

We write Comod- $H$ for the category of essential comodules over $H$ with $H$ colinear maps as morphisms. More precisely, we have defined right comodules. There are analogous definitions for left comodules. Let us point out that corepresentations and comodules in the framework of multiplier Hopf algebras have been discussed in detail in [van Daele and Zhang 1999; Kurose et al. 2000].

The most elementary example of a coaction is the trivial coaction $\tau$ of $H$ on $V$. The map $\tau: V \rightarrow \operatorname{Hom}_{H}(H, V \hat{\otimes} H)$ is given by $\tau(v)(f)=v \otimes f$. Equivalently, the linear map $V \hat{\otimes} H \rightarrow V \hat{\otimes} H$ corresponding to $\tau$ is the identity.

As in the case of essential modules, there exists a tensor product in the category of essential comodules. Assume that $\eta_{V}: V \hat{\otimes} H \rightarrow V \hat{\otimes} H$ and $\eta_{W}: W \hat{\otimes} H \rightarrow W \hat{\otimes} H$ are essential comodules. Then the tensor product coaction $\eta_{V \hat{\otimes} W}$ is defined as the composition

$$
V \hat{\otimes} W \hat{\otimes} H \stackrel{\eta_{W}^{23}}{\longrightarrow} V \hat{\otimes} W \hat{\otimes} H \stackrel{\eta_{V}^{13}}{\longrightarrow} V \hat{\otimes} W \hat{\otimes} H .
$$

It is clear that $\eta_{V \hat{\otimes} W}$ is a right $H$-linear isomorphism and a straightforward calculation shows that it is indeed a coaction. The trivial coaction on $\mathbb{C}$ behaves like a unit with respect to the tensor product of comodules.

An important example of a coaction is the regular coaction of $H$ on itself given by the comultiplication $\Delta: H \rightarrow M(H \hat{\otimes} H)$. More precisely, the regular coaction is the map from $H$ to $\operatorname{Hom}_{H}(H, H \hat{\otimes} H)$ corresponding to the Galois map $\gamma_{r}$. The relation

$$
\left(\mathrm{id} \otimes \gamma_{r}\right) \gamma_{r}^{12}\left(\mathrm{id} \otimes \gamma_{r}^{-1}\right)=\gamma_{r}^{12} \gamma_{r}^{13}
$$

is easily verified. Note that this corresponds to the pentagon equation of the KacTakesaki operator [Baaj and Skandalis 1993].

Consider the special case that the bornological quantum group $H$ is unital. Then there is a natural isomorphism $\operatorname{Hom}_{H}(H, V \hat{\otimes} H) \cong V \hat{\otimes} H$ and a coaction is the same thing as a bounded linear map $\eta: V \rightarrow V \hat{\otimes} H$ such that $(\eta \hat{\otimes} \operatorname{id}) \eta=(\operatorname{id} \hat{\otimes} \Delta) \eta$ and (id $\hat{\otimes} \epsilon) \eta=\mathrm{id}$. That is, for unital bornological quantum groups the notion of a coaction is very similar to the concept of a coaction as it is used in the theory of Hopf algebras. 
For later use we give the following definitions. An essential $H$-module $P$ over a bornological quantum group $H$ is called projective if for every $H$-linear map $\pi: V \rightarrow W$ with bounded linear splitting $\sigma: W \rightarrow V$ and every $H$-linear map $\xi: P \rightarrow W$ there exists an $H$-linear map $\zeta: P \rightarrow V$ such that $\pi \zeta=\xi$. In this case we say that $P$ satisfies the lifting property for linearly split surjections of $\mathrm{H}$ modules. In a completely analogous way one defines the notion of a projective essential $H$-comodule.

We conclude this section by studying the functoriality of essential modules and comodules under morphisms of quantum groups. Let $\alpha: H \rightarrow M(K)$ be a morphism of bornological quantum groups. If $\lambda: K \hat{\otimes} V \rightarrow V$ is an essential $K$-module structure on $V$ then $\alpha^{*}(\lambda)$ is the $H$-module structure defined by

$$
H \hat{\otimes} V \stackrel{\alpha \hat{\otimes} \mathrm{id}}{\longrightarrow} M(K) \hat{\otimes} V \stackrel{\cong}{\longrightarrow} M(K) \hat{\otimes} K \hat{\otimes}_{K} V \stackrel{\mu \hat{\otimes} \mathrm{id}}{\longrightarrow} K \hat{\otimes}_{K} V \cong V,
$$

and it is easy to check that $V$ becomes an essential $H$-module in this way. This construction is evidently compatible with module maps and thus yields a functor $\alpha^{*}: K$-Mod $\rightarrow H$-Mod. A similar functor is obtained for right modules.

Conversely, let $\eta: V \hat{\otimes} H \rightarrow V \hat{\otimes} H$ be an essential $H$-comodule. We define a bounded linear map $\alpha_{*}(\eta): V \hat{\otimes} K \rightarrow V \hat{\otimes} K$ by the commutative diagram

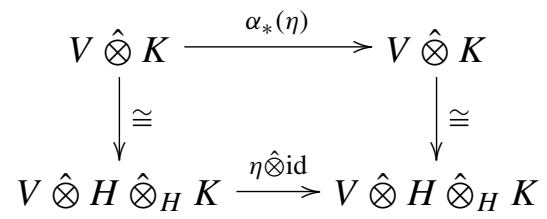

where we use that $\eta$ is right $H$-linear. It is evident that $\alpha_{*}(\eta)$ is a right $K$-linear isomorphism and one checks that the relation

$$
\left(\mathrm{id} \otimes \gamma_{r}\right) \alpha_{*}(\eta)_{12}\left(\mathrm{id} \otimes \gamma_{r}^{-1}\right)=\alpha_{*}(\eta)_{12} \alpha_{*}(\eta)_{13}
$$

is satisfied. Hence $\alpha_{*}(\eta)$ defines a coaction of $K$ on $V$. This construction is compatible with comodule maps and yields a functor $\alpha_{*}$ : Comod- $H \rightarrow$ Comod-K. Again, there is a similar functor for left comodules.

\section{The dual quantum group and Pontrjagin duality}

In this section we construct the dual quantum group $\hat{H}$ of a bornological quantum group $H$. Moreover we prove the analogue of Pontrjagin duality in the context of bornological quantum groups. Unless further specified we assume that $\phi$ is a left Haar functional on $H$ and we let $\psi$ be any right Haar functional. 
Using the invariant functional $\phi$ we define bounded linear maps $\mathscr{F}_{l}$ and $\mathscr{F}_{r}$ from $H$ into the dual space $H^{\prime}=\operatorname{Hom}(H, \mathbb{C})$ by

$$
\mathscr{F}_{l}(f)(h)=\phi(h f), \quad \mathscr{F}_{r}(f)(h)=\phi(f h) .
$$

Similarly, we obtain bounded linear maps $\mathscr{G}_{l}$ and $\mathscr{G}_{r}$ from $H$ into $H^{\prime}$ by

$$
\mathscr{G}_{l}(f)(h)=\psi(h f), \quad \varphi_{r}(f)(h)=\psi(f h),
$$

and all these maps are injective by faithfulness. Using notation and results from Section 5 we obtain:

Proposition 7.1. Let $H$ be a bornological quantum group. Then

$$
\mathscr{F}_{r}(f)=\mathscr{F}_{l}(\sigma(f)), \quad \mathscr{G}_{l}(f)=\mathscr{F}_{l}(v(f)), \quad \mathscr{G}_{r}(f)=\mathscr{\varphi}_{l}(\rho(f))
$$

for all $f \in H$.

Due to Proposition 7.1 the images of the maps $\mathscr{F}_{l}, \mathscr{F}_{r}, \mathscr{G}_{l}, \mathscr{G}_{r}$ in $H^{\prime}$ coincide. Let us write $\hat{H}$ for this space. Moreover, since the maps $\sigma, v$ and $\rho$ are isomorphisms we may use any of them to define a unique bornology on $\hat{H}$ by transferring the bornology from $H$. We will always view $\hat{H}$ as a bornological vector space with this bornology and hence the maps $\mathscr{F}_{l}, \mathscr{F}_{r}, \mathscr{G}_{l}, \mathscr{G}_{r}$ yield bornological isomorphisms from $H$ to $\hat{H}$. In particular, the space $\hat{H}$ satisfies again the approximation property.

We say that a bounded bilinear map $b: U \times V \rightarrow W$ is nondegenerate if $b(u, v)=$ 0 for all $u \in U$ implies $v=0$ and $b(u, v)=0$ for all $v \in V$ implies $u=0$. Since $H$ is a regular bornological vector space the canonical pairing between $H$ and $H^{\prime}$ given by $\langle f, \omega\rangle=\omega(f)$ is nondegenerate. By construction of the space $\hat{H}$ there is an obvious injective bounded linear map $\hat{H} \rightarrow H^{\prime}$ and we have a nondegenerate pairing between $H$ and $\hat{H}$ as well. The latter may be extended naturally to a pairing between $M(H)$ and $\hat{H}$ which is again nondegenerate. There are similar constructions for tensor powers of $H$ and $\hat{H}$.

In order to obtain a quantum group structure on $\hat{H}$ our first aim is to define a multiplication. Consider the transpose map $\Delta^{*}: M(H \hat{\otimes} H)^{\prime} \rightarrow H^{\prime}$ of the comultiplication given by

$$
\Delta^{*}(\omega)(f)=\omega(\Delta(f)) .
$$

According to the previous remarks, $\hat{H} \hat{\otimes} \hat{H}$ can be viewed as a linear subspace of $M(H \hat{\otimes} H)^{\prime}$ and $\Delta^{*}$ restricts to a map $\hat{H} \hat{\otimes} \hat{H} \rightarrow H^{\prime}$. We shall show that the latter actually yields a bounded linear map $\hat{H} \hat{\otimes} \hat{H} \rightarrow \hat{H}$. To do this we define a bounded linear map $m: H \hat{\otimes} H \rightarrow H$ by

$$
m(f \otimes g)=(\operatorname{id} \hat{\otimes} \phi) \gamma_{l}^{-1}(f \otimes g) .
$$

Transferring this map according to the isomorphism $\mathscr{F}_{l}: H \rightarrow \hat{H}$ we obtain a bounded linear map $\hat{\mu}: \hat{H} \hat{\otimes} \hat{H} \rightarrow \hat{H}$ which we call the convolution product. One 
computes

$(\mathrm{id} \hat{\otimes} \mu)\left(\mu \hat{\otimes} \operatorname{id}_{(2)}\right)\left(S \hat{\otimes} \operatorname{id}_{(3)}\right)(\operatorname{id} \hat{\otimes} \Delta \hat{\otimes} \mathrm{id})\left(\mu_{(2)} \hat{\otimes} \mathrm{id}\right)\left(\Delta \hat{\otimes} \operatorname{id}_{(3)}\right)$ $=(\operatorname{id} \hat{\otimes} \mu)\left(\mu \hat{\otimes} \operatorname{id}_{(2)}\right)\left(S \hat{\otimes} \operatorname{id}_{(3)}\right)\left(\operatorname{id} \hat{\otimes} \rho_{r} \hat{\otimes} \operatorname{id}\right)\left(\tau \hat{\otimes} \operatorname{id}_{(2)}\right)$,

and we conclude that

$(\mu \hat{\otimes} \mathrm{id})\left(S \hat{\otimes} \mathrm{id}_{(2)}\right)(\mathrm{id} \hat{\otimes} \Delta) \mu_{(2)}\left(\Delta \hat{\otimes} \mathrm{id}_{(2)}\right)=(\mu \hat{\otimes} \mathrm{id})\left(S \hat{\otimes} \mathrm{id}_{(2)}\right)\left(\mathrm{id} \hat{\otimes} \rho_{r}\right)(\tau \hat{\otimes} \mathrm{id})$. Moreover,

$$
\tau(\mu \hat{\otimes} \mathrm{id})\left(\mathrm{id} \hat{\otimes} \gamma_{l}^{-1}\right)=(\mu \hat{\otimes} \mathrm{id})(\tau \hat{\otimes} \mathrm{id})\left(\mathrm{id} \hat{\otimes} S^{-1} \hat{\otimes} \mathrm{id}\right)\left(\mathrm{id} \hat{\otimes} \rho_{r}\right)(\tau \hat{\otimes} \mathrm{id}) .
$$

Using invariance we compute

$$
\begin{aligned}
\hat{\mu}\left(\mathscr{F}_{l}(f) \otimes \mathscr{F}_{l}(g)\right)(h) & =(\phi \hat{\otimes} \phi)(\mu \hat{\otimes} \mathrm{id})\left(\mathrm{id} \hat{\otimes} \gamma_{l}^{-1}\right)(h \otimes f \otimes g) \\
& =\Delta^{*}\left(\mathscr{F}_{l}(f) \otimes \mathscr{F}_{l}(g)\right)(h)
\end{aligned}
$$

and deduce that $\hat{\mu}$ can be identified with $\Delta^{*}$. From this one calculates $\hat{\mu}(\hat{\mu} \hat{\otimes} \mathrm{id})\left(\mathscr{F}_{l}(f) \otimes \mathscr{F}_{l}(g) \otimes \mathscr{F}_{l}(h)\right)(x)=\hat{\mu}(\mathrm{id} \hat{\otimes} \hat{\mu})\left(\mathscr{F}_{l}(f) \otimes \mathscr{F}_{l}(g) \otimes \mathscr{F}_{l}(h)\right)(x)$, which means that the convolution product $\hat{\mu}$ is associative. Hence $\hat{H}$ is a bornological algebra with convolution as multiplication. According to the above considerations we have

$$
\hat{\mu}\left(\mathscr{F}_{l}(f) \otimes \mathscr{F}_{l}(g)\right)=\mathscr{F}_{l}\left((\mathrm{id} \hat{\otimes} \phi) \gamma_{l}^{-1}(f \otimes g)\right)
$$

and an analogous calculation yields the formula

$$
\hat{\mu}\left(\mathscr{G}_{r}(f) \otimes \mathscr{G}_{r}(g)\right)=\mathscr{G}_{r}\left((\psi \hat{\otimes} \mathrm{id}) \rho_{r}^{-1}(f \otimes g)\right)
$$

for the multiplication in $\hat{H}$. Actually, this equation can be obtained directly from the previous discussion applied to $H^{\text {op cop }}$.

For later use we shall extend the multiplication of $\hat{H}$ in the following way. From (4-18) and the fact that $\phi$ is left invariant we have

$$
(\mathrm{id} \hat{\otimes} \phi) \gamma_{r}=(\mathrm{id} \hat{\otimes} \phi) \gamma_{l} \tau\left(S^{-1} \hat{\otimes} \mathrm{id}\right) \rho_{r}=(\mathrm{id} \hat{\otimes} \phi)\left(S^{-1} \hat{\otimes} \mathrm{id}\right) \rho_{r} .
$$

Using this observation we define a bounded linear map $\hat{\mu}_{l}: H^{\prime} \hat{\otimes} \hat{H} \rightarrow H^{\prime}$ by

$$
\hat{\mu}_{l}\left(\omega \otimes \mathscr{F}_{l}(f)\right)(x)=(\omega \hat{\otimes} \phi) \gamma_{r}(x \otimes f)=(\omega \hat{\otimes} \phi)\left(S^{-1} \hat{\otimes} \mathrm{id}\right) \rho_{r}(x \otimes f) .
$$

Inserting $\omega=\epsilon$ we see that $\hat{\mu}_{l}\left(\omega \otimes \mathscr{F}_{l}(f)\right)=0$ for all $\omega$ implies $f=0$. Conversely, assume $\hat{\mu}_{l}\left(\omega \otimes \mathscr{F}_{l}(f)\right)=0$ for all $f \in H$. Then we have $(\omega \hat{\otimes} \phi) \gamma_{r}(h \otimes f)=0$ for all $h, f \in H$ and since $\gamma_{r}$ is an isomorphism this yields $\omega=0$. Hence $\hat{\mu}_{l}$ defines a nondegenerate pairing 
Similarly, according to (4-17) we have

$$
(\psi \hat{\otimes} \mathrm{id}) \rho_{l}=(\psi \hat{\otimes} \mathrm{id})\left(\mathrm{id} \hat{\otimes} S^{-1}\right) \gamma_{r}^{-1} \gamma_{l}=(\psi \hat{\otimes} \mathrm{id})\left(\mathrm{id} \hat{\otimes} S^{-1}\right) \gamma_{l}
$$

and we define $\hat{\mu}_{r}: \hat{H} \hat{\otimes} H^{\prime} \rightarrow H^{\prime}$ by

$$
\hat{\mu}_{r}\left(\varphi_{r}(f) \otimes \omega\right)(x)=(\psi \hat{\otimes} \omega) \rho_{l}(f \otimes x)=(\psi \hat{\otimes} \omega)\left(\mathrm{id} \hat{\otimes} S^{-1}\right) \gamma_{l}(f \otimes x) .
$$

As above one sees that the pairing given by $\hat{\mu}_{r}$ is nondegenerate. If restricted to $\hat{H} \hat{\otimes} \hat{H}$ the maps $\hat{\mu}_{l}$ and $\hat{\mu}_{r}$ are equal to the multiplication map $\hat{\mu}$. Moreover it is straightforward to check that the maps $\hat{\mu}_{l}$ and $\hat{\mu}_{r}$ are associative whenever this assertion makes sense. In the sequel we will simply write $\hat{\mu}$ for the maps $\hat{\mu}_{l}$ and $\hat{\mu}_{r}$, respectively.

Using the definition of the modular automorphism $\sigma$ we obtain

$$
(\mathrm{id} \hat{\otimes} \phi) \rho_{r}(f \otimes x)=(\operatorname{id} \hat{\otimes} \phi) \gamma_{r}(x \otimes \sigma(f)),
$$

and together with (7-3) this yields the formula

$$
\hat{\mu}\left(\omega \otimes \mathscr{F}_{r}(f)\right)(x)=(\omega \hat{\otimes} \phi) \rho_{r}(f \otimes x)=(\omega \hat{\otimes} \phi)(S \hat{\otimes} \mathrm{id}) \gamma_{r}(f \otimes x)
$$

for the multiplication $\hat{\mu}$. In a similar way we obtain

$$
\hat{\mu}\left(\mathscr{G}_{l}(f) \otimes \omega\right)(x)=(\psi \hat{\otimes} \omega) \gamma_{l}(x \otimes f)=(\psi \hat{\otimes} \omega)(\operatorname{id} \hat{\otimes} S) \rho_{l}(x \otimes f)
$$

using (7-5).

Our next aim is to show that $\hat{H}$ is a projective module over itself. In order to do this we study the regular coaction of $H$ on itself given by $\gamma_{r}$.

Proposition 7.2. The regular coaction of a bornological quantum group $H$ on itself is a projective $H$-comodule.

Proof. Choose an element $h \in H$ such that $\phi(h)=1$ and define $v: H \rightarrow H \hat{\otimes} H$ by $v(f)=\gamma_{l}(h \otimes f)$. Then we have

$$
(\mathrm{id} \hat{\otimes} \phi) v(f)=(\mathrm{id} \hat{\otimes} \phi) \gamma_{l}(h \otimes f)=\phi(h) f=f
$$

for all $f \in H$ since $\phi$ is left invariant. Hence the map $v$ satisfies the equation

$$
(\mathrm{id} \hat{\otimes} \phi) v=\text { id. }
$$

Let us moreover define $\lambda: H \rightarrow H \hat{\otimes} H$ by $\lambda=\gamma_{r}^{-1} \nu$

Now assume that $\pi: V \rightarrow W$ is a surjective map of $H$-comodules with bounded linear splitting $\sigma$ and let $\xi: H \rightarrow W$ be an $H$-colinear map. We define $\zeta: H \rightarrow V$ as the composition $\zeta=(\mathrm{id} \hat{\otimes} \phi) \eta_{V}(\sigma \xi \hat{\otimes} \mathrm{id}) \lambda$ where $\eta_{V}$ is the coaction of $V$. 
We check that $\zeta$ is $H$-colinear. Using Equation (4-17) we obtain $\left(\gamma_{r}^{-1} \hat{\otimes} \mathrm{id}\right)\left(\gamma_{l} \hat{\otimes} \mathrm{id}\right)\left(\mathrm{id} \hat{\otimes} \gamma_{r}\right)=(\mathrm{id} \hat{\otimes} S \hat{\otimes} \mathrm{id})\left(\rho_{l} \hat{\otimes} \mathrm{id}\right)\left(\mathrm{id} \hat{\otimes} \gamma_{r}\right)$

$$
=(\mathrm{id} \hat{\otimes} S \hat{\otimes} \mathrm{id})\left(\mathrm{id} \hat{\otimes} \gamma_{r}\right)\left(\mathrm{id} \hat{\otimes} S^{-1} \hat{\otimes} \mathrm{id}\right)\left(\gamma_{r}^{-1} \hat{\otimes} \mathrm{id}\right)\left(\gamma_{l} \hat{\otimes} \mathrm{id}\right)
$$

and deduce

$$
(\lambda \hat{\otimes} \mathrm{id}) \gamma_{r}=(\mathrm{id} \hat{\otimes} S \hat{\otimes} \mathrm{id})\left(\mathrm{id} \hat{\otimes} \gamma_{r}\right)\left(\mathrm{id} \hat{\otimes} S^{-1} \hat{\otimes} \mathrm{id}\right)(\lambda \hat{\otimes} \mathrm{id}) .
$$

Since $S$ is an algebra and coalgebra antihomomorphism we have $\tau(S \hat{\otimes} S) \gamma_{r}=$ $\rho_{l}(S \hat{\otimes} S) \tau$ which yields

$$
(S \hat{\otimes} \mathrm{id}) \gamma_{r}\left(S^{-1} \hat{\otimes} \mathrm{id}\right)=\tau\left(S^{-1} \hat{\otimes} \mathrm{id}\right) \rho_{l}(S \hat{\otimes} \mathrm{id}) \tau .
$$

Using Equations (4-18) and (4-20) we obtain

$$
\rho_{r}=(S \hat{\otimes} \mathrm{id}) \tau \gamma_{l}^{-1} \gamma_{r}=\rho_{l}(S \hat{\otimes} \mathrm{id}) \gamma_{r} .
$$

Since $\eta_{V}$ is right $H$-linear we calculate

$$
\eta_{V}^{13}\left(\mathrm{id} \hat{\otimes} \rho_{r}\right)(\mathrm{id} \hat{\otimes} \mu \hat{\otimes} \mathrm{id})=\left(\mathrm{id} \hat{\otimes} \rho_{r}\right) \eta_{V}^{12}(\mathrm{id} \hat{\otimes} \mu \hat{\otimes} \mathrm{id})
$$

which yields $\eta_{V}^{13}\left(\operatorname{id} \hat{\otimes} \rho_{r}\right)=\left(\operatorname{id} \hat{\otimes} \rho_{r}\right) \eta_{V}^{12}$ since $H$ is essential. Combining this with (7-12) implies

$$
\left(\operatorname{id} \hat{\otimes} \rho_{r}\right) \eta_{V}^{12}\left(\operatorname{id} \hat{\otimes} \gamma_{r}^{-1}\right)=\eta_{V}^{13}\left(\mathrm{id} \hat{\otimes} \rho_{l}\right)(\mathrm{id} \hat{\otimes} S \hat{\otimes} \mathrm{id}) .
$$

Using Equations (7-10), (7-11), (7-13) and (7-3) we calculate $(\zeta \hat{\otimes}$ id $) \gamma_{r}=\eta_{V}(\zeta \hat{\otimes}$ id) which shows that $\zeta$ is $H$-colinear

We now prove that $\zeta$ is a lifting for $\xi$. Since $\pi$ is colinear, the diagram

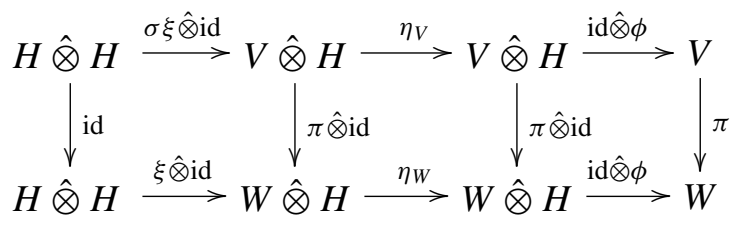

is commutative, and moreover $\eta_{W}(\xi \hat{\otimes} \mathrm{id})=(\xi \hat{\otimes} \mathrm{id}) \gamma_{r}$ because $\xi$ is colinear. As a consequence we get $\pi \zeta=\xi$ since

$$
(\mathrm{id} \hat{\otimes} \phi) \gamma_{r} \lambda=(\mathrm{id} \hat{\otimes} \phi) v=\mathrm{id}
$$

by the definition of $\lambda$.

Consider the transposed right regular coaction $\rho$ of $H$ on itself given by $\rho=$ $\gamma_{l}^{-1} \tau$. Since Equation (4-19) for $H^{\mathrm{op}}$ yields

$$
\gamma_{r}\left(S^{-1} \hat{\otimes} \mathrm{id}\right)=\left(S^{-1} \hat{\otimes} \mathrm{id}\right) \gamma_{l}^{-1} \tau
$$


we see that the transposed right regular coaction $\rho$ corresponds to the right regular coaction $\gamma_{r}$ under the linear automorphism of $H$ given by $S^{-1}$. It follows that the map $\rho$ is indeed a coaction and that the coactions $\rho$ and $\gamma_{r}$ yield isomorphic comodules. Hence the comodule defined by $\rho$ is projective due to Proposition 7.2

As above let $m: H \hat{\otimes} H \rightarrow H$ be the map corresponding to the multiplication of $\hat{H}$ under the isomorphism $\mathscr{F}_{l}$. By the definition of the right regular coaction we get

$$
m=(\mathrm{id} \hat{\otimes} \phi) \rho \tau \text {. }
$$

The pentagon relation for the operator $\gamma_{r}^{\text {cop }}=\tau \gamma_{l}=\rho^{-1}$ can be written as

$$
\rho^{23} \rho^{13} \rho^{12}=\rho^{12} \rho^{23} ;
$$

together with the formula (id $\hat{\otimes} \phi) \tau \rho^{-1}=(\operatorname{id} \otimes \phi) \gamma_{l}=(\operatorname{id} \hat{\otimes} \phi) \tau$ this implies

$$
(m \hat{\otimes} \mathrm{id}) \rho^{13}=(\mathrm{id} \hat{\otimes} \mathrm{id} \hat{\otimes} \phi)(\mathrm{id} \hat{\otimes} \tau)(\rho \tau \hat{\otimes} \mathrm{id}) \rho^{13},=\rho(m \otimes \mathrm{id})
$$

which means that the map $m$ is right $H$-colinear if we view $H \hat{\otimes} H$ as a right $H$ comodule using the coaction $\rho^{13}$. Since $m$ has a bounded linear splitting we obtain a colinear splitting $\sigma: H \rightarrow H \hat{\otimes} H$ due to Proposition 7.2. In other words, we have $(\sigma \hat{\otimes}$ id $) \rho=\rho^{13}(\sigma \hat{\otimes}$ id $)$, which yields

$\sigma m=\left(\operatorname{id}_{(2)} \hat{\otimes} \phi\right)(\sigma \hat{\otimes} \mathrm{id}) \rho \tau=(\mathrm{id} \hat{\otimes} \phi \hat{\otimes} \mathrm{id})(\rho \tau \hat{\otimes} \mathrm{id})(\mathrm{id} \hat{\otimes} \sigma)=(m \hat{\otimes} \mathrm{id})(\mathrm{id} \hat{\otimes} \sigma)$.

Translating this to $\hat{H}$ using the isomorphism $\mathscr{F}_{l}$ we see that there is a $\hat{H}$-linear splitting for the multiplication map $\hat{\mu}$ if $\hat{H}$ acts by multiplication on the left tensor factor of $\hat{H} \hat{\otimes} \hat{H}$. Using such a splitting it is straightforward to check that $\hat{H}$ is an essential bornological algebra.

We define a linear form $\hat{\psi}$ on $\hat{H}$ by $\hat{\psi}\left(\mathscr{F}_{l}(f)\right)=\epsilon(f)$ and compute

$$
\hat{\psi}\left(\mathscr{F}_{l}(f) \mathscr{F}_{l}(g)\right)=(\epsilon \hat{\otimes} \phi) \gamma_{l}^{-1}(f \otimes g)=\phi\left(S^{-1}(g) f\right)
$$

for all $f, g \in H$ which implies that $\hat{\psi}$ is faithful since $\phi$ is faithful. Hence the algebra $\hat{H}$ is equipped with a faithful bounded linear functional. We will see below that $\hat{\psi}$ is right invariant for the comultiplication of $\hat{H}$, but first, of course, we have to construct this comultiplication.

To do this we define a bounded linear map $\hat{\gamma}_{r}: \hat{H} \hat{\otimes} \hat{H} \rightarrow \hat{H} \hat{\otimes} \hat{H}$ by

$$
\left.\hat{\gamma}_{r}\left(\mathscr{F}_{l} l f\right) \otimes \mathscr{F}_{l}(g)\right)=\left(\mathscr{F}_{l} \hat{\otimes} \mathscr{F}_{l}\right) \tau \gamma_{l}^{-1}(f \otimes g) .
$$

It is evident that $\hat{\gamma}_{r}$ is a bornological isomorphism. Let us show that $\hat{\gamma}_{r}$ commutes with right multiplication on the second tensor factor. Using the pentagon relation (7-16) for $\rho$ and ( $\phi \hat{\otimes}$ id) $\rho=\phi \hat{\otimes}$ id we have

$(\mathrm{id} \hat{\otimes} \mathrm{id} \hat{\otimes} \phi)(\tau \hat{\otimes} \mathrm{id})\left(\gamma_{l}^{-1} \hat{\otimes} \mathrm{id}\right)\left(\mathrm{id} \hat{\otimes} \gamma_{l}^{-1}\right)(f \otimes g \otimes h)$

$$
=(\mathrm{id} \hat{\otimes} \mathrm{id} \hat{\otimes} \phi)\left(\mathrm{id} \hat{\otimes} \gamma_{l}^{-1}\right)(\tau \hat{\otimes} \mathrm{id})\left(\gamma_{l}^{-1} \hat{\otimes} \mathrm{id}\right)(f \otimes g \otimes h),
$$


and translating this using the map $\mathscr{F}_{l} l$ we obtain

$$
\hat{\gamma}_{r}(\mathrm{id} \hat{\otimes} \hat{\mu})=(\operatorname{id} \hat{\otimes} \hat{\mu})\left(\hat{\gamma}_{r} \hat{\otimes} \mathrm{id}\right),
$$

as desired. Similarly, we define a bornological automorphism $\hat{\rho}_{l}$ of $\hat{H} \hat{\otimes} \hat{H}$ by

$$
\hat{\rho}_{l}\left(\mathscr{G}_{r}(g) \otimes \mathscr{G}_{r}(f)\right)=\left(\mathscr{G}_{r} \hat{\otimes} \mathscr{G}_{r}\right) \tau \rho_{r}^{-1}(g \otimes f) .
$$

and using formula (7-2) we obtain

$$
\hat{\rho}_{l}(\hat{\mu} \hat{\otimes} \mathrm{id})=(\hat{\mu} \hat{\otimes} \mathrm{id})\left(\mathrm{id} \hat{\otimes} \hat{\rho}_{l}\right) .
$$

From (7-3) and (7-5) we have

$(7-19) \hat{\gamma}_{r}\left(\omega \otimes \mathscr{F}_{l}(g)\right)(x \otimes y)=(\omega \hat{\otimes} \phi)(\mu \hat{\otimes} \mathrm{id})\left(\mathrm{id} \hat{\otimes} S^{-1} \hat{\otimes} \mathrm{id}\right)\left(\mathrm{id} \hat{\otimes} \rho_{r}\right)(x \otimes y \otimes g)$ and

$(7-20) \hat{\rho}_{l}\left(\varphi_{r}(f) \otimes \omega\right)(x \otimes y)=(\psi \hat{\otimes} \omega)(\mathrm{id} \hat{\otimes} \mu)\left(\mathrm{id} \hat{\otimes} S^{-1} \hat{\otimes} \mathrm{id}\right)\left(\gamma_{l} \hat{\otimes} \mathrm{id}\right)(f \otimes x \otimes y)$ for all $\omega \in \hat{H}$. Using (7-4) and (7-6) we calculate $(\mathrm{id} \hat{\otimes} \hat{\mu})\left(\hat{\rho}_{l} \hat{\otimes} \mathrm{id}\right)\left(\mathscr{G}_{r}(f) \otimes \omega \otimes \mathscr{F}_{l}(g)\right)(x \otimes y)$

$$
=(\hat{\mu} \hat{\otimes} \mathrm{id})\left(\mathrm{id} \hat{\otimes} \hat{\gamma}_{r}\right)\left(\mathscr{G}_{r}(f) \otimes \omega \otimes \mathscr{F}_{l}(g)\right)(x \otimes y),
$$

which yields

$$
(\mathrm{id} \hat{\otimes} \hat{\mu})\left(\hat{\rho}_{l} \hat{\otimes} \mathrm{id}\right)=(\hat{\mu} \hat{\otimes} \mathrm{id})\left(\mathrm{id} \hat{\otimes} \hat{\gamma}_{r}\right) .
$$

Using the pentagon relation (7-16) for $\rho$ we compute $\hat{\gamma}_{r}(\hat{\mu} \hat{\otimes} \mathrm{id})\left(\mathscr{F}_{l}(f) \otimes \mathscr{F}_{l}(g) \otimes \mathscr{F}_{l}(h)\right)$

$$
=(\hat{\mu} \hat{\otimes} \mathrm{id})(\tau \hat{\otimes} \mathrm{id})\left(\mathrm{id} \hat{\otimes} \hat{\gamma}_{r}\right)(\tau \hat{\otimes} \mathrm{id})\left(\mathrm{id} \hat{\otimes} \hat{\gamma}_{r}\right)\left(\mathscr{F}_{l}(f) \otimes \mathscr{F}_{l}(g) \otimes \mathscr{F}_{l}(h)\right),
$$

which gives

$$
\hat{\gamma}_{r}(\hat{\mu} \hat{\otimes} \mathrm{id})=(\hat{\mu} \hat{\otimes} \mathrm{id})(\tau \hat{\otimes} \mathrm{id})\left(\mathrm{id} \hat{\otimes} \hat{\gamma}_{r}\right)(\tau \hat{\otimes} \mathrm{id})\left(\mathrm{id} \hat{\otimes} \hat{\gamma}_{r}\right) .
$$

A similar computation shows that

$$
\hat{\rho}_{l}(\mathrm{id} \hat{\otimes} \hat{\mu})=(\operatorname{id} \hat{\otimes} \hat{\mu})(\mathrm{id} \hat{\otimes} \tau)\left(\hat{\rho}_{l} \hat{\otimes} \mathrm{id}\right)(\mathrm{id} \hat{\otimes} \tau)\left(\hat{\rho}_{l} \hat{\otimes} \mathrm{id}\right) .
$$

Finally, we have

$\left(\hat{\rho}_{l} \hat{\otimes} \mathrm{id}\right)\left(\mathrm{id} \hat{\otimes} \hat{\gamma}_{r}\right)\left(\varphi_{r}(f) \otimes \omega \otimes \mathscr{F}_{l}(g)\right)(x \otimes y \otimes z)$

$$
=\left(\mathrm{id} \hat{\otimes} \hat{\gamma}_{r}\right)\left(\hat{\rho}_{l} \hat{\otimes} \mathrm{id}\right)\left(\varphi_{r}(f) \otimes \omega \otimes \mathscr{F}_{l}(g)\right)(x \otimes y \otimes z),
$$

according to (7-19) and (7-20), and hence

$$
\left(\hat{\rho}_{l} \hat{\otimes} \mathrm{id}\right)\left(\mathrm{id} \hat{\otimes} \hat{\gamma}_{r}\right)=\left(\mathrm{id} \hat{\otimes} \hat{\gamma}_{r}\right)\left(\hat{\rho}_{l} \hat{\otimes} \mathrm{id}\right) .
$$


Using the properties of the maps $\hat{\gamma}_{r}$ and $\hat{\rho}_{l}$ obtained so far we shall construct the comultiplication for $\hat{H}$ according to the following general result.

Proposition 7.3. Let $K$ be an essential bornological algebra satisfying the approximation property equipped with a faithful bounded linear functional. If $\gamma_{r}$ and $\rho_{l}$ are bornological automorphisms of $K \hat{\otimes} K$ such that

(a) $\gamma_{r}($ id $\hat{\otimes} \mu)=($ id $\hat{\otimes} \mu)\left(\gamma_{r} \hat{\otimes}\right.$ id $)$,

(b) $\gamma_{r}(\mu \hat{\otimes} \mathrm{id})=(\mu \hat{\otimes} \mathrm{id})(\tau \hat{\otimes} \mathrm{id})\left(\mathrm{id} \hat{\otimes} \gamma_{r}\right)(\tau \hat{\otimes} \mathrm{id})\left(\mathrm{id} \hat{\otimes} \gamma_{r}\right)$,

(c) $\rho_{l}(\mu \hat{\otimes} \mathrm{id})=(\mu \hat{\otimes} \mathrm{id})\left(\mathrm{id} \hat{\otimes} \rho_{l}\right)$,

(d) $\rho_{l}(\mathrm{id} \hat{\otimes} \mu)=(\mathrm{id} \hat{\otimes} \mu)(\mathrm{id} \hat{\otimes} \tau)\left(\rho_{l} \hat{\otimes} \mathrm{id}\right)(\mathrm{id} \hat{\otimes} \tau)\left(\rho_{l} \hat{\otimes} \mathrm{id}\right)$,

(e) $(\mathrm{id} \hat{\otimes} \mu)\left(\rho_{l} \hat{\otimes} \mathrm{id}\right)=(\mu \hat{\otimes} \mathrm{id})\left(\mathrm{id} \hat{\otimes} \gamma_{r}\right)$,

(f) $\left(\rho_{l} \hat{\otimes} \mathrm{id}\right)\left(\mathrm{id} \hat{\otimes} \gamma_{r}\right)=\left(\mathrm{id} \hat{\otimes} \gamma_{r}\right)\left(\rho_{l} \hat{\otimes} \mathrm{id}\right)$,

then there exists a unique comultiplication $\Delta: K \rightarrow M(K \hat{\otimes} K)$ such that $\gamma_{r}$ and $\rho_{l}$ are the associated Galois maps.

If there exist also bornological automorphisms $\gamma_{l}$ and $\rho_{r}$ of $K \hat{\otimes} K$ such that

(g) $(\mu \hat{\otimes} \mathrm{id})(\mathrm{id} \hat{\otimes} \tau)\left(\gamma_{l} \hat{\otimes} \mathrm{id}\right)=\gamma_{l}(\mathrm{id} \hat{\otimes} \mu)$,

(h) $(\mathrm{id} \hat{\otimes} \mu)\left(\gamma_{l} \hat{\otimes} \mathrm{id}\right)=(\mu \hat{\otimes} \mathrm{id})(\mathrm{id} \hat{\otimes} \tau)\left(\gamma_{r} \hat{\otimes} \mathrm{id}\right)(\mathrm{id} \hat{\otimes} \tau)$,

(i) $(\mathrm{id} \hat{\otimes} \mu)(\tau \hat{\otimes} \mathrm{id})\left(\mathrm{id} \hat{\otimes} \rho_{r}\right)=\rho_{r}(\mu \hat{\otimes} \mathrm{id})$,

(j) $(\mu \hat{\otimes} \mathrm{id})\left(\mathrm{id} \hat{\otimes} \rho_{r}\right)=(\mathrm{id} \hat{\otimes} \mu)(\tau \hat{\otimes} \mathrm{id})\left(\mathrm{id} \hat{\otimes} \rho_{l}\right)(\tau \hat{\otimes} \mathrm{id})$,

then these maps are the remaining Galois maps. Thus all Galois maps are isomorphisms in this case.

Proof. Using condition a) it is straightforward to check that

$$
\mu_{(2)}\left(\Delta_{l} \hat{\otimes} \mathrm{id}_{(2)}\right)=(\mu \hat{\otimes} \mathrm{id})(\mathrm{id} \hat{\otimes} \tau)\left(\gamma_{r} \hat{\otimes} \mathrm{id}\right)(\mathrm{id} \hat{\otimes} \tau)
$$

defines a bounded linear map $\Delta_{l}: K \rightarrow M_{l}(K \hat{\otimes} K)$. According to condition b) the map $\Delta_{l}$ is actually a homomorphism. Similarly,

$$
\mu_{(2)}\left(\operatorname{id}_{(2)} \hat{\otimes} \Delta_{r}\right)=(\operatorname{id} \hat{\otimes} \mu)(\tau \hat{\otimes} \mathrm{id})\left(\mathrm{id} \hat{\otimes} \rho_{l}\right)(\tau \hat{\otimes} \mathrm{id})
$$

defines a homomorphism $\Delta_{r}: K \rightarrow M_{r}(K \hat{\otimes} K)$ due to conditions c) and d). Condition e) ensures that these maps combine to an algebra homomorphism $\Delta$ : $K \rightarrow M(K \hat{\otimes} K)$. It is straightforward to show that $\gamma_{r}$ and $\rho_{l}$ are the corresponding Galois maps. Moreover $\Delta$ is uniquely determined by these maps

We have to prove that the homomorphism $\Delta$ is essential. Let us show that the natural map $K \hat{\otimes}_{K}(K \hat{\otimes} K) \rightarrow K \hat{\otimes} K$ is an isomorphism where the module structure on $K \hat{\otimes} K$ is given by $\Delta$. Since $K$ is essential we have $K \hat{\otimes}_{K} K \cong K$ and we can 
identify the source of the previous map with $K \hat{\otimes}_{K}\left(K \hat{\otimes}\left(K \hat{\otimes}_{K} K\right)\right)$ in a natural way. It is easy to check that $\gamma_{r}^{13}$ descends to a bounded linear map

$$
\xi: K \hat{\otimes}_{K}\left(K \hat{\otimes}\left(K \hat{\otimes}_{K} K\right)\right) \rightarrow\left(K \hat{\otimes}_{K} K\right) \hat{\otimes}\left(K \hat{\otimes}_{K} K\right)
$$

and the composition of $\xi$ with $\mu \hat{\otimes} \mu$ can be identified with the map we are interested in. Hence it suffices to show that $\xi$ is an isomorphism. Consider the maps

$$
\begin{aligned}
& p=\mu \hat{\otimes} \mathrm{id} \hat{\otimes} \mu \hat{\otimes} \mathrm{id}-\left(\mathrm{id} \hat{\otimes} \mu \hat{\otimes} \mathrm{id}_{(2)}\right) \gamma_{r}^{24}\left(\operatorname{id}_{(4)} \hat{\otimes} \mu\right), \\
& q=\mu \hat{\otimes} \mathrm{id} \hat{\otimes} \mu \hat{\otimes} \mathrm{id}-\mathrm{id} \hat{\otimes} \mu \hat{\otimes} \mathrm{id} \hat{\otimes} \mu,
\end{aligned}
$$

defined on the six-fold tensor product of $K$ with itself. The source and target of $\xi$ are the quotients of $K^{\hat{\otimes} 4}$ by the closure of the image of $p$ and $q$, respectively. Using conditions a) and b) it is straightforward to verify the relation $q \kappa=\gamma_{r}^{13} p$ where $\kappa$ is the bornological automorphism of $K^{\hat{\otimes} 6}$ defined by

$$
\kappa=\left(\operatorname{id}_{(2)} \hat{\otimes} \tau \hat{\otimes} \operatorname{id}_{(2)}\right) \gamma_{r}^{13} \gamma_{r}^{23}\left(\operatorname{id}_{(2)} \hat{\otimes} \tau \hat{\otimes} \operatorname{id}_{(2)}\right) .
$$

This relation shows that $\xi$ is actually a bornological isomorphism. Using the map $\rho_{l}$ one proves in a similar way that $(K \hat{\otimes} K) \hat{\otimes}_{K} K \rightarrow K \hat{\otimes} K$ is an isomorphism. We conclude that $\Delta$ is essential. Having established this, condition $\mathrm{f}$ ) immediately yields that $\Delta$ is coassociative. Hence $\Delta$ is a comultiplication.

If there exists maps $\gamma_{l}$ and $\rho_{r}$ with the properties stated in conditions g) and h) then these maps describe the remaining Galois maps associated to $\Delta$. It follows in particular that all Galois maps yield isomorphisms from $K \hat{\otimes} K$ into itself in this case.

We have already shown above that the maps $\hat{\gamma}_{r}$ and $\hat{\rho}_{l}$ satisfy the assumptions of Proposition 7.3. Let us write $\hat{\Delta}$ for the comultiplication on $\hat{H}$ defined in this way. By construction, the Galois maps $\hat{\gamma}_{r}$ and $\hat{\rho}_{l}$ associated to $\hat{\Delta}$ are isomorphisms.

To treat the remaining Galois maps for $\hat{\Delta}$, we define abstractly

$$
\begin{gathered}
\hat{\gamma}_{l}\left(\mathscr{F}_{r}(f) \otimes \mathscr{F}_{r}(g)\right)=\left(\mathscr{F}_{r} \hat{\otimes} \mathscr{F}_{r}\right) \tau \rho_{l}^{-1}(f \otimes g), \\
\hat{\rho}_{r}\left(\mathscr{G}_{l}(g) \otimes \mathscr{G}_{l}(f)\right)=\left(\mathscr{G}_{l} \hat{\otimes} \mathscr{G}_{l}\right) \tau \gamma_{r}^{-1}(g \otimes f) .
\end{gathered}
$$

Applying the preceding discussion to $H^{\text {op }}$ we see that $\hat{\gamma}_{l}$ and $\hat{\rho}_{r}$ satisfy conditions (g) and (i) in Proposition 7.3. Using (7-3) and (7-5) it is straightforward to obtain the formulas

$$
\hat{\gamma}_{l}\left(\omega \otimes \mathscr{F}_{r}(g)\right)(x \otimes y)=(\omega \hat{\otimes} \phi)(\mu \hat{\otimes} \mathrm{id})(\mathrm{id} \hat{\otimes} \tau)\left(\rho_{r} \hat{\otimes} \mathrm{id}\right)(g \otimes x \otimes y)
$$

and

$$
\hat{\rho}_{r}\left(\mathscr{G}_{l}(f) \otimes \omega\right)(x \otimes y)=(\psi \hat{\otimes} \omega)(\mathrm{id} \hat{\otimes} \mu)(\tau \hat{\otimes} \mathrm{id})\left(\mathrm{id} \hat{\otimes} \gamma_{l}\right)(x \otimes y \otimes f)
$$


for the maps $\hat{\gamma}_{l}$ and $\hat{\rho}_{r}$. Using the definition of $\hat{\mu}$ and Equations (7-25), (7-3) and (7-19), we compute

$(\mathrm{id} \hat{\otimes} \hat{\mu})\left(\hat{\gamma}_{l} \hat{\otimes} \mathrm{id}\right)\left(\omega \otimes \mathscr{F}_{r}(f) \otimes \mathscr{F}_{l}(g)\right)(x \otimes y)$

$$
\begin{aligned}
& =\left(\hat{\gamma}_{l}\left(\omega \otimes \mathscr{F}_{r}(f)\right) \hat{\otimes} \phi\right)\left(x \otimes \gamma_{r}(y \otimes g)\right) \\
& =(\hat{\mu} \hat{\otimes} \mathrm{id})(\mathrm{id} \hat{\otimes} \tau)\left(\hat{\gamma}_{r} \hat{\otimes} \mathrm{id}\right)(\mathrm{id} \hat{\otimes} \tau)\left(\omega \otimes \mathscr{F}_{r}(f) \otimes \mathscr{F}_{l}(g)\right)(x \otimes y),
\end{aligned}
$$

which yields condition (h). Using Equations (7-26), (7-5) and (7-20) one obtains condition (j) in a similar way. Hence it follows from Proposition 7.3 that all Galois maps associated to $\hat{\Delta}$ are isomorphisms.

It remains to exhibit the Haar functionals for the comultiplication $\hat{\Delta}$. The proof of the following proposition is straightforward.

Proposition 7.4. Let $H$ be a bornological quantum group. Then the linear form $\hat{\psi}$ on $\hat{H}$ defined by

$$
\hat{\psi}\left(\mathscr{F}_{l}(f)\right)=\epsilon(f)
$$

is a faithful right invariant functional on $\hat{H}$. Similarly, the linear form $\hat{\phi}$ given by

$$
\hat{\phi}\left(\varphi_{r}(f)\right)=\epsilon(f)
$$

is a faithful left invariant functional on $\hat{H}$.

We have now completed to proof of the following theorem.

Theorem 7.5. Let $H$ be a bornological quantum group. Then $\hat{H}$ with the structure maps described above is again a bornological quantum group.

The bornological quantum group $\hat{H}$ will be called the dual quantum group of $H$. It is instructive to describe explicitly the counit and the antipode of $\hat{H}$. Consider the map $\hat{\epsilon}: \hat{H} \rightarrow \mathbb{C}$ given by

$$
\hat{\epsilon}(\omega)=\omega(1),
$$

where $\hat{H}$ is viewed as a subspace of $M(H)^{\prime}$ according to the nondegenerate pairing $\hat{H} \times M(H) \rightarrow \mathbb{C}$. The explicit formulas

$\hat{\epsilon}\left(\mathscr{F}_{l}(f)\right)=\phi(f), \quad \hat{\epsilon}\left(\mathscr{F}_{r}(f)\right)=\phi(f), \quad \hat{\epsilon}\left(\mathscr{G}_{l}(f)\right)=\psi(f), \quad \hat{\epsilon}\left(\mathscr{G}_{r}(f)\right)=\psi(f)$

show that the map $\hat{\epsilon}$ is bounded and nonzero. It is straightforward to check that $\hat{\epsilon}$ is an algebra homomorphism and we calculate

$$
\begin{aligned}
(\hat{\epsilon} \hat{\otimes} \mathrm{id}) \hat{\gamma}_{r}\left(\mathscr{F}_{l}(f) \otimes \mathscr{F}_{l}(h)\right) & =\left(\mathscr{F}_{l} \hat{\otimes} \phi\right) \gamma_{l}^{-1}(f \otimes h)=\hat{\mu}\left(\mathscr{F}_{l}(f) \otimes \mathscr{F}_{l}(h)\right), \\
(\mathrm{id} \hat{\otimes} \hat{\epsilon}) \hat{\rho}_{l}\left(\mathscr{G}_{r}(h) \otimes \mathscr{G}_{r}(f)\right) & =\left(\psi \hat{\otimes} \mathscr{G}_{r}\right) \rho_{r}^{-1}(h \otimes f)=\hat{\mu}\left(\mathscr{G}_{r}(h) \otimes \mathscr{G}_{r}(f)\right),
\end{aligned}
$$

which shows $(\hat{\epsilon} \hat{\otimes} \mathrm{id}) \gamma_{r}=\hat{\mu}$ and $(\mathrm{id} \hat{\otimes} \hat{\epsilon}) \rho_{l}=\hat{\mu}$. One can then proceed as in the proof of Theorem 4.6 to show that $\hat{\epsilon}$ is nondegenerate. By the uniqueness assertion of Theorem 4.6 we see that the map $\hat{\epsilon}$ is indeed the counit for $\hat{H}$. 
Similarly, we define $\hat{S}: \hat{H} \rightarrow \hat{H}$ by $\hat{S}(\omega)(f)=\omega(S(f))$, and using $\psi=S(\phi)$ we obtain the formulas

$$
\hat{S}\left(\mathscr{F}_{l}(f)\right)=\mathscr{G}_{r}\left(S^{-1}(f)\right), \quad \hat{S}\left(\mathscr{F}_{r}(f)\right)=\mathscr{G}_{l}\left(S^{-1}(f)\right) .
$$

It follows that $\hat{S}$ is a bounded linear automorphism of $\hat{H}$. Using (7-3) we compute

$$
\hat{\mu}(\hat{S} \hat{\otimes} \mathrm{id})\left(\mathscr{F}_{l}(f) \otimes \mathscr{F}_{l}(g)\right)(x)=(\hat{\epsilon} \hat{\otimes} \mathrm{id}) \hat{\gamma}_{r}^{-1}\left(\mathscr{F}_{l}(f) \otimes \mathscr{F}_{l}(g)\right)(x),
$$

which shows $\hat{\mu}(\hat{S} \hat{\otimes} \mathrm{id})=(\hat{\epsilon} \hat{\otimes} \mathrm{id}) \hat{\gamma}_{r}^{-1}$. In a similar way one obtains the relation $\hat{\mu}(\operatorname{id} \hat{\otimes} \hat{S})=(\operatorname{id} \hat{\otimes} \hat{\epsilon}) \hat{\rho}_{l}^{-1}$. Inspecting the constructions in the proof of Theorem 4.6 we see that $\hat{S}$ is the antipode of $\hat{H}$.

Theorem 7.6 (Pontrjagin duality theorem). Let $H$ be a bornological quantum group. Then the double dual quantum group of $H$ is canonically isomorphic to $H$.

Proof. We define a linear map $P: H \rightarrow(\hat{H})^{\prime}$ by $P(f)(\omega)=\omega(f)$ for all $f \in H$ and $\omega \in \hat{H}$. Using Proposition 7.4 we compute

$$
\begin{aligned}
\left(\hat{\mathscr{G}}_{l} \mathscr{F}_{l}(f)\right)\left(\mathscr{F}_{l}(h)\right) & =\hat{\psi}\left(\mathscr{F}_{l}(h) \mathscr{F}_{l}(f)\right)=\hat{\psi}\left(\mathscr{F}_{l} \hat{\otimes} \phi\right) \gamma_{l}^{-1}(f \otimes h) \\
& =(\epsilon \hat{\otimes} \phi) \gamma_{l}^{-1}(f \otimes h)=\phi\left(S^{-1}(f) h\right)=\mathscr{F}_{l}(h)\left(S^{-1}(f)\right)
\end{aligned}
$$

for all $f, g \in H$, where $\hat{\mathscr{G}}_{l}$ is the map $\mathscr{G}_{l}$ for $\hat{H}$. This implies

$$
P(f)=\hat{\mathscr{G}}_{l} \mathscr{F}_{l}(S(f))
$$

and shows that $P$ defines a bornological isomorphism from $H$ to $\hat{\hat{H}}$. In a similar way one has $P(f)=\hat{\mathscr{F}}_{r} \varphi_{r}(S(f))$. Further, using $\psi=S^{-1}(\phi)$ one calculates

$$
\begin{aligned}
\left(\hat{\mathscr{F}}_{l} S \mathscr{G}_{l}(f)\right)\left(\mathscr{G}_{r}(h)\right) & =\hat{\phi}\left(\mathscr{G}_{r}(h) \mathscr{F}_{r}\left(S^{-1}(f)\right)\right) \\
& =(\phi \hat{\otimes} \epsilon) \rho_{r}^{-1}\left(h \otimes S^{-1}(f)\right)=\mathscr{G}_{r}(h)(f),
\end{aligned}
$$

which shows $P=\hat{\mathscr{F}}_{l} S \varphi_{l}$.

Next consider the transpose $\mu^{*}: H^{\prime} \rightarrow(H \hat{\otimes} H)^{\prime}$ of the multiplication map, which is given by

$$
\mu^{*}(\omega)(f \otimes g)=\omega \mu(f \otimes g)=\omega(f g)
$$

for all $f, g \in H$. In particular, we obtain a bounded linear map $\mu^{*}: \hat{H} \rightarrow(H \hat{\otimes} H)^{\prime}$ by restriction. Using the isomorphism $P$ we can view $\hat{\Delta}$ as a map from $\hat{H} \rightarrow(H \hat{\otimes}$ $H)^{\prime}$ as well. Equivalently, we have bounded linear maps from $\hat{H}$ into $\operatorname{Hom}\left(H, H^{\prime}\right)$ given by

$$
\mu^{*}(\omega)(g)(f)=\omega \mu(f \otimes g)
$$


and likewise for $\hat{\Delta}$. Using (7-14) we calculate

$$
\hat{\Delta}\left(\mathscr{F}_{l}(h)\right)(g)=(\mathrm{id} \hat{\otimes} \hat{\psi}) \hat{\gamma}_{r}\left(\mathscr{F}_{l}(h) \otimes \mathscr{F}_{l} S(g)\right)=\mathscr{F}_{l}(g h)
$$

and thus obtain, using the definition of $S^{-1}$,

$$
\begin{aligned}
\hat{\Delta}\left(\mathscr{F}_{l}(h)\right)(g)(f) & =\hat{\psi}\left(\mathscr{F}_{l}(g h) \mathscr{F}_{l} S(f)\right)=\hat{\psi}\left(\mathscr{F}_{l} \hat{\otimes} \phi\right) \gamma_{l}^{-1}(g h \otimes S(f)) \\
& =(\epsilon \hat{\otimes} \phi) \gamma_{l}^{-1}(g h \otimes S(f))=\phi(f g h)=\mu^{*}\left(\mathscr{F}_{l}(h)\right)(g)(f),
\end{aligned}
$$

which shows that $\hat{\Delta}$ can be identified with the transpose $\mu^{*}$ of the multiplication. Similarly, we have seen in the constructions above that $\hat{\mu}$ can be identified with the transpose $\Delta^{*}$ of the comultiplication.

With this in mind it is easy to check that $P$ is an algebra homomorphism and a coalgebra homomorphism. Hence $P$ is an isomorphism of bornological quantum groups.

\section{Duality for modules and comodules}

In this section we study the duality between essential modules and comodules over a bornological quantum group and its dual

Let $H$ be a bornological quantum group and let $\eta: V \rightarrow \operatorname{Hom}_{H}(H, V \hat{\otimes} H)$ be an essential $H$-comodule. We define a bounded linear map $D(\eta): \hat{H} \hat{\otimes} V \rightarrow V$ by

$$
D(\eta)\left(\mathscr{F}_{l}(f) \otimes v\right)=(\mathrm{id} \hat{\otimes} \phi) \eta(v \otimes f) .
$$

For later use we need another description of this map. Since $H$ is an essential algebra we may view $\eta$ as a bounded linear map from $V$ into $\operatorname{Hom}_{H}(H, V \hat{\otimes} H) \cong$ $\operatorname{Hom}_{H}\left(H \hat{\otimes}_{H} H, V \hat{\otimes} H\right)$. Under the latter isomorphism $\eta(v)$ corresponds to the map $(\operatorname{id} \hat{\otimes} \mu)(\eta(v) \hat{\otimes}$ id). Moreover, using notation and results from Section 5 we have

$$
\phi(h g v(f))=\psi(h g f)=\phi(h v(g f))
$$

for all $f, g, h \in H$ which implies that $v$ is left $H$-linear. Together with the relation

$$
(\mathrm{id} \hat{\otimes} \phi)(\operatorname{id} \hat{\otimes} \mu)(\eta(v)(g) \otimes v(f))=(\operatorname{id} \hat{\otimes} \psi)(\operatorname{id} \hat{\otimes} \mu)(\eta(v)(g) \otimes f)
$$

we thus obtain $D(\eta)\left(\varphi_{l}(f) \otimes v\right)=(\mathrm{id} \hat{\otimes} \psi) \eta(v \otimes f)$. Next we compute

$$
\begin{aligned}
D(\eta)(\mathrm{id} \hat{\otimes} D(\eta))\left(\mathscr{F}_{l}(f) \otimes \mathscr{F}_{l}(g)\right. & \otimes v) \\
= & (\mathrm{id} \hat{\otimes} \phi) \eta\left(D(\eta)\left(\mathscr{F}_{l}(g) \otimes v\right) \otimes f\right) \\
= & (\operatorname{id} \hat{\otimes} \phi \hat{\otimes} \phi)\left(\mathrm{id} \otimes \gamma_{r}\right) \eta_{12}\left(\mathrm{id} \otimes \gamma_{r}^{-1}\right)(v \otimes f \otimes g) .
\end{aligned}
$$

From (7-3) we have $(\phi \hat{\otimes} \phi) \gamma_{r}=(\phi \hat{\otimes} \phi)\left(S^{-1} \hat{\otimes}\right.$ id $) \rho_{r}$, and using (4-18) and (7-13) we obtain

$$
D(\eta)(\mathrm{id} \hat{\otimes} D(\eta))\left(\mathscr{F}_{l}(f) \otimes \mathscr{F}_{l}(g) \otimes v\right)=D(\eta)(\hat{\mu} \hat{\otimes} \mathrm{id})\left(\mathscr{F}_{l}(f) \otimes \mathscr{F}_{l}(g) \otimes v\right),
$$


which shows that $V$ becomes a left $\hat{H}$-module in this way.

We want to show that $V$ is actually an essential $\hat{H}$-module. In order to do this it is convenient to work with the map $(\operatorname{id} \hat{\otimes} \phi) \eta$ instead of $D(\eta)$. There is an evident bounded linear splitting $\sigma: V \rightarrow V \hat{\otimes} H$ of this map given by $\sigma(v)=\eta^{-1}(v \otimes h)$ where $h$ is chosen such that $\phi(h)=1$. If we identify $\hat{H} \hat{\otimes}_{\hat{H}} V$ accordingly with a quotient $Q$ of $V \hat{\otimes} H$ we have the relation

$$
(\mathrm{id} \hat{\otimes} \mathrm{id} \hat{\otimes} \phi) \eta_{13}=(\mathrm{id} \hat{\otimes} \mathrm{id} \hat{\otimes} \phi)\left(\mathrm{id} \hat{\otimes} \gamma_{l}^{-1}\right)
$$

in this quotient. Now we see, as in the proof of Proposition 7.2 and using formula (7-14), that

$$
(\mathrm{id} \hat{\otimes} \mathrm{id} \hat{\otimes} \phi) \eta_{12} \eta_{13}=(\mathrm{id} \hat{\otimes} \phi \hat{\otimes} \mathrm{id}) \eta_{12}\left(\mathrm{id} \otimes \gamma_{l}^{-1}\right),
$$

which implies that

$$
\eta^{-1}(\mathrm{id} \hat{\otimes} \phi \hat{\otimes} \mathrm{id}) \eta_{12}=(\mathrm{id} \hat{\otimes} \mathrm{id} \hat{\otimes} \phi) \eta_{13}\left(\mathrm{id} \hat{\otimes} \gamma_{l}\right)=\mathrm{id} \hat{\otimes} \mathrm{id} \hat{\otimes} \phi
$$

in $Q$. It follows that $\sigma(\mathrm{id} \otimes \phi) \eta$ is the identity map on $Q$. Translating this back to $\hat{H} \hat{\otimes}_{\hat{H}} V$ we deduce that $V$ is an essential module.

An $H$-colinear map $f: V \rightarrow W$ is easily seen to be $\hat{H}$-linear for the module structures defined in this way. Hence we have proved the following statement.

Proposition 8.1. Let $H$ be a bornological quantum group and let $\hat{H}$ be the dual quantum group. The previous construction defines a functor $D$ from Comod- $H$ to $\hat{H}$-Mod.

Conversely, let $\lambda: H \hat{\otimes} V \rightarrow V$ be an essential left $H$-module. By slight abuse of notation we write $\lambda^{-1}$ for the inverse of the isomorphism $H \hat{\otimes}_{H} V \cong V$ induced by $\lambda$. We define a bounded linear map $D(\lambda): V \hat{\otimes} \hat{H} \rightarrow V \hat{\otimes} \hat{H}$ by

$$
D(\lambda)\left(v \otimes \mathscr{F}_{l}(f)\right)=\left(\operatorname{id} \hat{\otimes} \mathscr{F}_{l}\right) \tau(\mathrm{id} \hat{\otimes} \lambda)\left(\gamma_{l}^{-1} \tau \hat{\otimes} \mathrm{id}\right)\left(\mathrm{id} \hat{\otimes} \lambda^{-1}\right)(f \otimes v),
$$

which is seen to be well-defined since $\gamma_{l}^{-1} \tau$ is right $H$-linear for the action by multiplication on the second tensor factor. It is evident that $D(\lambda)$ is an isomorphism. Since $\lambda$ is left $H$-linear we calculate, with $\rho=\gamma_{l}^{-1} \tau$,

$(\mathrm{id} \hat{\otimes} \lambda)\left(\gamma_{l}^{-1} \tau \hat{\otimes} \mathrm{id}\right)\left(\mathrm{id} \hat{\otimes} \lambda^{-1}\right)(\mathrm{id} \hat{\otimes} \phi \hat{\otimes} \mathrm{id})\left(\gamma_{l}^{-1} \hat{\otimes} \mathrm{id}\right)(f \otimes g \otimes v)$

$$
=(\mathrm{id} \hat{\otimes} \phi \hat{\otimes} \mathrm{id})\left(\gamma_{l}^{-1} \hat{\otimes} \mathrm{id}\right)(\tau \hat{\otimes} \lambda)\left(\mathrm{id} \hat{\otimes} \gamma_{l}^{-1} \tau \hat{\otimes} \mathrm{id}\right)\left(\tau \hat{\otimes} \lambda^{-1}\right)(f \otimes g \otimes v),
$$

using $(\phi \hat{\otimes} \mathrm{id})=(\phi \hat{\otimes} \mathrm{id}) \rho$ as well as the pentagon relation (7-16) for the map $\rho$. This shows that

$$
D(\lambda)(\mathrm{id} \hat{\otimes} \hat{\mu})\left(v \otimes \mathscr{F}_{l}(f) \otimes \mathscr{F}_{l}(g)\right)=(\mathrm{id} \hat{\otimes} \hat{\mu})(D(\lambda) \hat{\otimes} \mathrm{id})\left(v \otimes \mathscr{F}_{l}(f) \otimes \mathscr{F}_{l}(g)\right),
$$


which means that $D(\lambda)$ is right $\hat{H}$-linear. Again by the pentagon relation for $\rho$ and the $H$-linearity of $\lambda$ is we have

$$
\begin{aligned}
& (\tau \hat{\otimes} \lambda)\left(\mathrm{id} \hat{\otimes} \gamma_{l}^{-1} \tau \hat{\otimes} \mathrm{id}\right)\left(\tau \hat{\otimes} \mathrm{id}_{(2)}\right)\left(\mathrm{id} \hat{\otimes} \gamma_{l}^{-1} \tau \hat{\otimes} \mathrm{id}\right)\left(\operatorname{id}_{(2)} \hat{\otimes} \lambda^{-1}\right)\left(\tau \gamma_{l}^{-1} \hat{\otimes} \mathrm{id}\right)(f \otimes g \otimes v) \\
& =\left(\tau \gamma_{l}^{-1} \hat{\otimes} \mathrm{id}\right)(\tau \hat{\otimes} \mathrm{id})\left(\mathrm{id}_{(2)} \hat{\otimes} \lambda\right)\left(\mathrm{id} \hat{\otimes} \gamma_{l}^{-1} \tau \hat{\otimes} \mathrm{id}\right)\left(\tau \hat{\otimes} \mathrm{id}_{(2)}\right)\left(\mathrm{id}_{(2)} \hat{\otimes} \lambda^{-1}\right)(f \otimes g \otimes v),
\end{aligned}
$$

which shows that

$$
D(\lambda)_{12} D(\lambda)_{13}\left(\mathrm{id} \hat{\otimes} \hat{\gamma}_{r}\right)(v \otimes \mathscr{F}(f) \otimes \mathscr{F}(g))=\left(\mathrm{id} \hat{\otimes} \hat{\gamma}_{r}\right) D(\lambda)_{12}(v \otimes \mathscr{F}(f) \otimes \mathscr{F}(g)) .
$$

Hence $D(\lambda)$ is a right coaction of $\hat{H}$ on $V$. It is easy to check that an $H$-equivariant map $f: V \rightarrow W$ between $H$-modules defines an $\hat{H}$-colinear map between the associated comodules.

Proposition 8.2. Let $H$ be a bornological quantum group and let $\hat{H}$ be the dual quantum group. There is a natural functor from $H$-Mod to Comod- $\hat{H}$ which will again be denoted by $D$.

Theorem 8.3 (Duality theorem for modules and comodules). Let $H$ be a bornological quantum group. Every essential left $H$-module is an essential right $\hat{H}$ comodule in a natural way and vice versa. This yields inverse isomorphisms between the category of essential $H$-modules and the category of essential $\hat{H}$ comodules. These isomorphisms are compatible with tensor products.

Of course an analogous statement holds for right modules and left comodules. Proof. We check that the functors defined above are inverse to each other if we take into account the Pontrjagin duality Theorem 7.6. By Equation (4-20) we have $\gamma_{l}^{-1}=\tau\left(S^{-1} \hat{\otimes} \mathrm{id}\right) \rho_{l}(S \hat{\otimes} \mathrm{id})$ and hence

$$
(\epsilon \hat{\otimes} \mathrm{id}) \gamma_{l}^{-1} \tau(S \hat{\otimes} \mathrm{id})=(\mathrm{id} \hat{\otimes} \epsilon)\left(S^{-1} \hat{\otimes} \mathrm{id}\right) \rho_{l}(S \hat{\otimes} S) \tau=S^{-1} \mu(S \hat{\otimes} S) \tau=\mu .
$$

Using the definition of the right Haar functional $\hat{\psi}$ on $\hat{H}$ we thus compute for an essential $H$-module $\lambda: H \hat{\otimes} V \rightarrow V$

$(\operatorname{id} \hat{\otimes} \hat{\psi}) D(\lambda)\left(v \otimes \mathscr{F}_{l}(S(f))\right)=\lambda(\operatorname{id} \hat{\otimes} \lambda)\left(\operatorname{id} \hat{\otimes} \lambda^{-1}\right)(f \otimes v)=\lambda(f \otimes v)$.

Consequently we have

$$
D D(\lambda)\left(\hat{G}_{l} \mathscr{F}_{l}(S(f)) \otimes v\right)=\lambda(f \otimes v)
$$

and according to Pontrjagin duality this shows that the module structure $D D(\lambda)$ can be identified with $\lambda$

Conversely, let $\eta: V \rightarrow \operatorname{Hom}_{H}(V, V \hat{\otimes} H)$ be an essential $H$-comodule. Using (7-14) for $\hat{H}$ we compute

$$
\begin{aligned}
D D(\eta)\left(v \otimes \hat{\mathscr{F}}_{l} S(\omega)\right) & =\left(\operatorname{id} \hat{\otimes} \hat{\mathscr{F}}_{l}\right) \tau(\operatorname{id} \hat{\otimes} D(\eta))\left(\hat{\gamma}_{l}^{-1} \tau \hat{\otimes} \mathrm{id}\right)\left(\operatorname{id} \hat{\otimes} D(\eta)^{-1}\right)(S(\omega) \otimes v) \\
& =\tau\left(\hat{\mathscr{F}}_{l} S \hat{\otimes} \mathrm{id}\right)(\operatorname{id} \hat{\otimes} D(\eta))\left(\hat{\gamma}_{r} \hat{\otimes} \mathrm{id}\right)\left(\operatorname{id} \hat{\otimes} D(\eta)^{-1}\right)(\omega \otimes v),
\end{aligned}
$$


and thus obtain

$$
D D(\eta)\left(D(\eta)\left(\mathscr{F}_{l}(g) \otimes v\right) \otimes \hat{\mathscr{F}}_{l} S \varphi_{l}(f)\right)=\left(\operatorname{id} \hat{\otimes} \hat{\mathscr{F}}_{l} S \varphi_{l}\right) \eta\left(D(\eta)\left(\mathscr{F}_{l}(g) \otimes v\right) \otimes f\right),
$$

which implies

$$
D D(\eta)\left(v \otimes \hat{\mathscr{F}}_{l} S \varphi_{l}(f)\right)=\left(\mathrm{id} \hat{\otimes} \hat{\mathscr{F}}_{l} S \varphi_{l}\right) \eta(v \otimes f)
$$

since $D(\eta)$ is an essential $\hat{H}$-module. Again by Pontrjagin duality this shows that $D D(\eta)$ is isomorphic to $\eta$

Consider $H \hat{\otimes}_{H}\left(H \hat{\otimes}\left(H \hat{\otimes}_{H} H\right)\right)$ and $\left(H \hat{\otimes}_{H} H\right) \hat{\otimes}\left(H \hat{\otimes}_{H} H\right)$ as $H$-modules by multiplication on the first tensor factor and by the diagonal action on the first and third tensor factors, respectively. Then the isomorphism $\xi$ used in the proof of Proposition 7.3 is $H$-linear. Using this it is straightforward to check that the functor $D$ from $H$-Mod to Comod- $H$ is compatible with tensor products.

We now use this duality result to construct the dual of a morphism between bornological quantum groups.

Proposition 8.4. Let $\alpha: H \rightarrow M(K)$ be a morphism of bornological quantum groups. Then there exists a unique morphism $\hat{\alpha}: \hat{K} \rightarrow M(\hat{H})$ such that

$$
\langle\alpha(f), \omega\rangle=\langle f, \hat{\alpha}(\omega)\rangle
$$

for all $f \in H$ and $\omega \in \hat{K}$.

Proof. Uniqueness of $\hat{\alpha}$ follows immediately from the nondegeneracy of the pairing between $H$ and $M(\hat{H})$. Consider the transposed right regular coaction $\rho=\gamma_{l}^{-1} \tau$ on $H$. The dual action of the pushforward coaction $\alpha_{*}(\rho)$ yields a left $\hat{K}$-module structure on $H$. Using the linear isomorphism $\mathscr{F}_{l}$ we may view this as a $\hat{K}$-module structure on $\hat{H}$. Associativity of the multiplication in $\hat{H}$ and (7-15) shows that we obtain in fact a bounded linear map $\hat{\alpha}_{l}: \hat{K} \rightarrow M_{l}(\hat{H})$. Similarly, the map $\gamma=\rho_{r}^{-1} \tau$ defines a left coaction of $H$ on itself, and the dual action of the corresponding pushforward coaction determines a right $\hat{K}$-module structure on $H$. This action yields a homomorphism $\hat{\alpha}_{r}: \hat{K} \rightarrow M(\hat{H})$. Using Lemma 4.5 for $H^{\text {cop }}$ we obtain

$$
(\mathrm{id} \hat{\otimes} \rho)(\gamma \hat{\otimes} \mathrm{id})=(\gamma \hat{\otimes} \mathrm{id})(\mathrm{id} \hat{\otimes} \rho),
$$

so the resulting left and right $\hat{K}$-module structures on $\hat{H}$ commute. Hence the maps $\hat{\alpha}_{l}$ and $\hat{\alpha}_{r}$ yield a nondegenerate homomorphism $\hat{\alpha}: \hat{K} \rightarrow M(\hat{H})$.

Consider also the transpose $\alpha^{*}: \hat{K} \rightarrow H^{\prime}$ of $\alpha$ given by $\alpha^{*}(\omega)(f)=\omega(\alpha(f))$. Then we have $\left\langle f, \alpha^{*}(\omega)\right\rangle=\langle\alpha(f), \omega\rangle$. Moreover the calculation after (4-16) for $H^{\text {cop }}$ gives

$$
(\mu \hat{\otimes} \mathrm{id})(\mathrm{id} \hat{\otimes} \rho)=(\mathrm{id} \hat{\otimes} \mu)\left(\mathrm{id} \hat{\otimes} S^{-1} \hat{\otimes} \mathrm{id}\right)\left(\tau \rho_{r} \hat{\otimes} \mathrm{id}\right) .
$$


Using (7-3), the definition of $\hat{\alpha}_{l}$ an that of $\hat{\mu}: H^{\prime} \hat{\otimes} \hat{H} \rightarrow H^{\prime}$ we calculate

$$
\hat{\mu}\left(\hat{\alpha}_{l} \hat{\otimes} \mathrm{id}\right)\left(\mathscr{F}_{l}(k) \otimes \mathscr{F}_{l}(f)\right)(h)=\hat{\mu}\left(\alpha^{*} \hat{\otimes} \mathrm{id}\right)\left(\mathscr{F}_{l}(k) \hat{\otimes} \mathscr{F}_{l}(f)\right)(h),
$$

where $\lambda_{l}$ denotes the isomorphism $H \hat{\otimes}_{H} K \cong K$ induced by $\alpha$. This shows

$$
\hat{\mu}\left(\hat{\alpha}_{l}\left(\mathscr{F}_{l}(k)\right) \otimes \mathscr{F}_{l}(f)\right)=\hat{\mu}\left(\alpha^{*}\left(\mathscr{F}_{l}(k)\right) \otimes \mathscr{F}_{l}(f)\right)
$$

for all $k \in K$ and $f \in H$. Similarly we have

$$
\left.\left.\hat{\mu}\left(\mathscr{G}_{r}(f)\right) \otimes \hat{\alpha}_{r}\left(\mathscr{G}_{r}(k)\right)\right)=\hat{\mu}\left(\mathscr{G}_{r}(f)\right) \otimes \alpha^{*}\left(\mathscr{G}_{r}(k)\right)\right)
$$

and we obtain $\hat{\alpha}(\omega)=\alpha^{*}(\omega)$ for all $\omega \in \hat{K}$

We shall only sketch how to show that $\hat{\alpha}$ is a coalgebra homomorphism. Using (7-3) one obtains

$$
\begin{aligned}
(\phi \hat{\otimes} \phi) \mu_{(2)}\left(\rho_{l} \hat{\otimes} \operatorname{id}_{(2)}\right) & =(\phi \hat{\otimes} \phi)(\mu \hat{\otimes} \mathrm{id})(\mathrm{id} \hat{\otimes} \mu \hat{\otimes} \mathrm{id}) \gamma_{r}^{24} \\
& =(\phi \hat{\otimes} \phi) \mu_{(2)}\left(\operatorname{id}_{(2)} \hat{\otimes} \tau \gamma_{l}^{-1}\right),
\end{aligned}
$$

which shows that

$$
\left\langle\rho_{l}(f \otimes g), \mathscr{F}_{l}(h) \otimes \mathscr{F}_{l}(k)\right\rangle=\left\langle f \otimes g, \hat{\gamma}_{r}\left(\mathscr{F}_{l}(h) \otimes \mathscr{F}_{l}(k)\right)\right\rangle
$$

for all $f, g, h, k \in H$. This relation extends to the case where $f$ and $g$ are multipliers of $H$ and we have similar statements involving other Galois maps. Based on this we calculate

$$
\left\langle f \otimes g,(\hat{\alpha} \hat{\otimes} \hat{\alpha}) \hat{\gamma}_{r}\left(\mathscr{F}_{l}(k) \otimes \mathscr{F}_{l}(l)\right)\right\rangle=\left\langle f \otimes g, \hat{\gamma}_{r}(\hat{\alpha} \hat{\otimes} \hat{\alpha})\left(\mathscr{F}_{l}(k) \otimes \mathscr{F}_{l}(l)\right)\right\rangle
$$

and deduce $(\hat{\alpha} \hat{\otimes} \hat{\alpha}) \hat{\gamma}_{r}=\hat{\gamma}_{r}(\hat{\alpha} \hat{\otimes} \hat{\alpha})$, which easily implies that $\hat{\alpha}$ is compatible with the comultiplication.

\section{Bornological quantum groups associated to Lie groups}

In this section we describe a dual pair of bornological quantum groups associated naturally to every Lie group. These bornological quantum groups are generalizations of the Hopf algebra of functions $C(G)$ and the group algebra $\mathbb{C} G$ of a finite group $G$. We will indicate at the end of this section how the constructions described below can be extended to arbitrary locally compact groups

If $M$ is a smooth manifold we let $\mathscr{D}(M)$ be the space of smooth functions on $M$ with compact support. The space $\mathscr{D}(M)$ is equipped with the bornology associated to its natural LF-topology. The following assertion is immediate.

Lemma 9.1. Let $M$ be a smooth manifold. The multiplier algebra of the algebra $\mathscr{D}(M)$ of smooth functions with compact support with pointwise multiplication is the algebra $\mathscr{E}(M)$ of all smooth functions. 
Now let $G$ be a Lie group. We choose a left Haar measure $d t$ and denote the modular function of $G$ by $\delta$. Let us write $C_{c}^{\infty}(G)$ for the bornological algebra of smooth functions on $G$ with pointwise multiplication. Using Lemma 9.1 one defines the comultiplication $\Delta: C_{c}^{\infty}(G) \rightarrow M\left(C_{c}^{\infty}(G \times G)\right)$ by

$$
\Delta(f)(r, s)=f(r s) .
$$

Proposition 9.2. Let $G$ be a Lie group. Then the algebra $C_{c}^{\infty}(G)$ of smooth functions with compact support on $G$ is a bornological quantum group.

Proof. It is straightforward to check that all Galois maps associated to $\Delta$ are isomorphisms. A left invariant integral $\phi$ for $C_{c}^{\infty}(G)$ is given by integration with respect to the Haar measure.

The counit $\epsilon: C_{c}^{\infty}(G) \rightarrow \mathbb{C}$ is given by $\epsilon(f)=f(e)$ where $e$ is the unit element of $G$. The antipode $S: C_{c}^{\infty}(G) \rightarrow C_{c}^{\infty}(G)$ is defined by $S(f)(t)=f\left(t^{-1}\right)$. The modular element in $M\left(C_{c}^{\infty}(G)\right)$ is given by the modular function $\delta$.

We describe the dual of $C_{c}^{\infty}(G)$. We write $\mathscr{D}(G)$ for this bornological quantum group and refer to it as the smooth group algebra of $G$. The underlying bornological vector space is of course again the space of smooth functions with compact support on $G$. Multiplication is given by the convolution product

$$
(f * g)(t)=\int_{G} f(s) g\left(s^{-1} t\right) d s
$$

which turns $\mathscr{D}(G)$ into a bornological algebra. Note that $\mathscr{D}(G)$ does not have a unit unless $G$ is discrete. The corresponding multiplier algebra is determined in [Meyer 2004b].

Proposition 9.3. Let $G$ be a Lie group. The multiplier algebra of the smooth group algebra $\mathscr{D}(G)$ is the algebra $\mathscr{E}^{\prime}(G)$ of distributions on $G$ with compact support.

We remark that the complex group ring $\mathbb{C} G$ is contained in $M(\mathscr{D}(G))=\mathscr{E}^{\prime}(G)$ as the subalgebra spanned by the Dirac distributions $\delta_{s}$ for $s \in G$.

Using Proposition 9.3 one describes the comultiplication $\Delta: \mathscr{D}(G) \rightarrow \mathscr{E}^{\prime}(G \times G)$ by

$$
\Delta(f)(h)=\int_{G} f(s) h(s, s) d s .
$$

The counit $\epsilon: \mathscr{D}(G) \rightarrow \mathbb{C}$ is defined by

$$
\epsilon(f)=\int_{G} f(s) d s,
$$

and the antipode $S: \mathscr{D}(G) \rightarrow \mathscr{D}(G)$ is given by $S(f)(t)=\delta(t) f\left(t^{-1}\right)$. The general theory developed in the previous sections yields immediately the following result.

Proposition 9.4. Let $G$ be a Lie group. Then the smooth group algebra $\mathscr{D}(G)$ of $G$ is a bornological quantum group. 
A left and right invariant integral $\phi$ for $\mathscr{D}(G)$ is given by $\phi(f)=f(e)$ where $e$ is the identity element

As mentioned above, one may as well consider smooth functions on arbitrary locally compact groups $G$ and obtain corresponding bornological quantum groups $C_{c}^{\infty}(G)$ and $\mathscr{D}(G)$. The definition of the space of smooth functions in this setting involves the structure theory of locally compact groups. More information can be found in [Meyer 2004b] where smooth representations of locally compact groups on bornological vector spaces are studied

Actually, it is immediate from the definitions that a smooth representation of the group $G$ is the same thing as an essential comodule over $C_{c}^{\infty}(G)$. In [Meyer 2004b] it is shown that the category of smooth representations of $G$ is naturally isomorphic to the category of essential modules over $\mathscr{D}(G)$. This result is a special case of Theorem 8.3 and explains the motivation for the general definitions of essential modules and comodules in Section 6.

\section{Schwartz algebras and discrete groups}

In this section we describe bornological quantum groups arising from Schwartz algebras of certain Lie groups as well as from algebras of functions satisfying various decay conditions on finitely generated discrete groups.

We begin with the abelian Lie group $G=\mathbb{R}^{n}$. Let $\mathscr{Y}\left(\mathbb{R}^{n}\right)$ be the Schwartz space of rapidly decreasing smooth functions on $\mathbb{R}^{n}$. The topology of this nuclear Fréchet space is defined by the seminorms

$$
p_{\alpha}^{k}(f)=\sup _{x \in \mathbb{R}^{n}}\left|\frac{\partial^{\alpha} f(x)}{\partial x_{\alpha}}(1+|x|)^{k}\right|
$$

for any multiindex $\alpha$ and any nonnegative integer $k$. Here $|x|$ denotes the euclidean norm of $x$. The space $\mathscr{Y}\left(\mathbb{R}^{n}\right)$ becomes an essential bornological algebra with the pointwise multiplication of functions. In order to identify the corresponding multiplier algebra recall that a function $f \in C^{\infty}\left(\mathbb{R}^{n}\right)$ is called slowly increasing if for every multiindex $\alpha$ there exists an integer $k$ such that

$$
\sup _{x \in \mathbb{R}^{n}}\left|\frac{1}{(1+|x|)^{k}} \frac{\partial^{\alpha} f(x)}{\partial x_{\alpha}}\right|<\infty .
$$

Slowly increasing functions on $\mathbb{R}^{n}$ form an algebra under pointwise multiplication. In fact:

Lemma 10.1. The multiplier algebra $M\left(\hat{\mathscr{Y}}\left(\mathbb{R}^{n}\right)\right)$ is the algebra of slowly increasing functions on $\mathbb{R}^{n}$.

To define the quantum group structure of $\mathscr{Y}\left(\mathbb{R}^{n}\right)$ the formulas for $C_{c}^{\infty}\left(\mathbb{R}^{n}\right)$ carry over. 
Proposition 10.2. The algebra $\mathscr{Y}\left(\mathbb{R}^{n}\right)$ of rapidly decreasing functions on $\mathbb{R}^{n}$ is a bornological quantum group.

We describe also the dual of $\mathscr{S}\left(\mathbb{R}^{n}\right)$. We will denote this quantum group by $\mathscr{S}^{*}\left(\mathbb{R}^{n}\right)$ and call it the tempered group algebra of $\mathbb{R}^{n}$. The underlying algebra structure is given by $\mathscr{S}\left(\mathbb{R}^{n}\right)$ with convolution multiplication. In order to determine the multiplier algebra of $\mathscr{S}^{*}\left(\mathbb{R}^{n}\right)$ let us denote by $B\left(\mathbb{R}^{n}\right)$ the space of all smooth functions $f$ on $\mathbb{R}^{n}$ such that all derivatives of $f$ are bounded. The topology on $B\left(\mathbb{R}^{n}\right)$ is given by uniform convergence of all derivatives. By definition, a bounded distribution is a continuous linear form on the space $B\left(\mathbb{R}^{n}\right)$. A distribution $T \in$ $\mathscr{D}^{\prime}\left(\mathbb{R}^{n}\right)$ has rapid decay if the distribution $T(1+|x|)^{k}$ is bounded for all $k \geq 0$. Using Fourier transform on obtains the following statement.

Lemma 10.3. The multiplier algebra $M\left(\mathscr{Y}\left(\mathbb{R}^{n}\right)\right)$ of $\mathscr{S}^{*}\left(\mathbb{R}^{n}\right)$ is the algebra of distributions with rapid decay.

The comultiplication, counit, antipode and the Haar integral for $\mathscr{S}^{*}\left(\mathbb{R}^{n}\right)$ can be determined in the same way as for the smooth group algebra $\mathscr{D}\left(\mathbb{R}^{n}\right)$. Remark that the classical Fourier transform can be viewed as an isomorphism of bornological quantum groups $\mathscr{S}^{*}\left(\mathbb{R}^{n}\right) \cong \mathscr{Y}\left(\hat{\mathbb{R}}^{n}\right)$ where $\hat{\mathbb{R}}^{n}$ is the dual group of $\mathbb{R}^{n}$.

The tempered group algebra $\mathscr{S}^{*}(\mathbb{R})$ and its dual as well as corresponding crossed products have been considered by Elliot, Natsume and Nest in [Elliott et al. 1988].

We remark that the abelian case treated above can be extended easily to simply connected nilpotent Lie groups. The algebra $\mathscr{S}(G)$ of Schwartz functions on a nilpotent Lie group $G$ has been considered by Natsume and Nest [1994] in connection with their study of the cyclic cohomology of the Heisenberg group.

Now let $\Gamma$ be a finitely generated discrete group equipped with a word metric. We denote by $L$ the associated length function on $\Gamma$. The function $L$ satisfies

$$
L(e)=0, \quad L(t)=L\left(t^{-1}\right) \quad \text { and } \quad L(s t) \leq L(s)+L(t)
$$

for all $s, t \in \Gamma$. Following [Meyer 2006] we define several function spaces associated to $\Gamma$. For every $k \in \mathbb{R}$ consider the norm

$$
p^{k}(f)=\sum_{t \in \Gamma}|f(t)|(1+L(t))^{k}
$$

on the complex group ring $\mathbb{C} \Gamma$ and denote by $\varphi^{k}(\Gamma)$ the corresponding Banach space completion. We write also $l^{1}(\Gamma)$ instead of $\mathscr{S}^{0}(\Gamma)$. Moreover let $\mathscr{S}(\Gamma)$ be the completion of $\mathbb{C} \Gamma$ with respect to the family of norms $p^{k}$ for all $k \in \mathbb{N}$. The natural map $\mathscr{S}^{k+1}(\Gamma) \rightarrow S^{k}(\Gamma)$ is compact for all $k \in \mathbb{N}$ and hence $\mathscr{S}(\Gamma)$ is a Fréchet Schwartz space. We call $\mathscr{S}(\Gamma)$ the space of Schwartz functions on $\Gamma$. 
Consider moreover the norm

$$
p_{\alpha}(f)=\sum_{t \in \Gamma}|f(t)| \alpha^{L(t)}
$$

for $\alpha>1$. We write $l^{1}(\Gamma, \alpha)$ for the completion of $\mathbb{C} \Gamma$ with respect to this norm and $\mathcal{O}(\Gamma)$ for the completion with respect to the family $p_{n}$ for $n \in \mathbb{N}$. Moreover let $\mathscr{S}^{\omega}(\Gamma)$ be the direct limit of the Banach spaces $l^{1}(\Gamma, \alpha)$ for $\alpha>1$. All these function spaces do not depend on the choice of the word metric.

All function spaces considered above become bornological algebras with the convolution product. The comultiplication, counit, antipode and the Haar functional of $\mathbb{C} \Gamma$ extend continuously to the completions.

Proposition 10.4. Let $\Gamma$ be a finitely generated discrete group. Then the algebras $l^{1}(\Gamma), \mathscr{Y}(\Gamma), \mathcal{O}(\Gamma)$ and $\mathscr{S}^{\omega}(\Gamma)$ are bornological quantum groups in a natural way.

The algebra structure of the corresponding dual quantum groups is obtained by equipping the above spaces of functions with pointwise multiplication.

\section{Rieffel deformation}

Rieffel [1993a] studied deformation quantization for Poisson brackets arising from actions of $\mathbb{R}^{d}$. Although the main focus in that monograph is on the study of the $C^{*}$-algebras arising in this way, a large part of the theory is carried out in the setting of Fréchet spaces.

If the underlying manifold is a Lie group, one may restrict attention to deformations compatible with the group structure. As it turns out, one obtains quantum groups in the setting of $C^{*}$-algebras in this way [Rieffel 1993b; 1995]. We shall only consider the case of compact Lie groups. A remarkable feature of the corresponding compact quantum groups is that they arise from deformations of the algebra of all smooth functions and not only of the algebra of representative functions. It is clear from the work of Rieffel that the deformed algebras of smooth functions fit naturally into the framework of bornological quantum groups.

Let $G$ be a compact Lie group and let $T$ be an $n$-dimensional torus in $G$ with Lie algebra $\mathfrak{t}$. We identify $\mathfrak{t}$ with $\mathbb{R}^{n}$ and set $V=\mathbb{R}^{n} \times \mathbb{R}^{n}$. Let exp $: \mathfrak{t} \rightarrow T$ denote the exponential map. Moreover let $J$ be a skew-symmetric operator on $V$ with respect to the standard inner product. In order to obtain a Poisson bracket which is compatible with the group structure of $G$ we shall assume that the operator $J$ is of the form $J=K \oplus(-K)$ where $K$ is a skew-symmetric operator on $\mathbb{R}^{n}$

Using this data, the deformed product of $f, g \in C^{\infty}(G)$ is defined by

$$
\left(f \star_{K} g\right)(x)=\int f(\exp (-K s) x \exp (-K u)) g(\exp (-t) x \exp (v)) e^{2 \pi i(\langle s, t\rangle+\langle u, v\rangle)},
$$


where the variables of integration range over $\mathbb{R}^{n}$. This yields a continuous and associative multiplication on $C^{\infty}(G)$, and we write $C^{\infty}(G)_{K}$ for the corresponding bornological algebra. Together with the classical comultiplication, antipode, counit and Haar integral of $C^{\infty}(G)$ this algebra becomes a bornological quantum group

We summarize this as follows and refer to [Rieffel 1993a, 1993b] for examples.

Proposition 11.1. Let $G$ be a compact Lie group and let $T$ be a torus in $G$ with Lie algebra $\mathfrak{t}$. For every skew-symmetric matrix $K$ on $\mathfrak{t}$ there exists a bornological quantum group $C^{\infty}(G)_{K}$ with structure as described above.

\section{References}

[Baaj and Skandalis 1993] S. Baaj and G. Skandalis, "Unitaires multiplicatifs et dualité pour les produits croisés de $C^{*}$-algèbres”, Ann. Sci. École Norm. Sup. (4) 26:4 (1993), 425-488. MR 94e:46127 Zbl 0804.46078

[van Daele 1994] A. van Daele, "Multiplier Hopf algebras", Trans. Amer. Math. Soc. 342:2 (1994), 917-932. MR 94h:16075 Zbl 0809.16047

[van Daele 1998] A. van Daele, "An algebraic framework for group duality", Adv. Math. 140:2 (1998), 323-366. MR 2000g:16045 Zbl 0933.16043

[van Daele and Zhang 1999] A. van Daele and Y. Zhang, "Corepresentation theory of multiplier Hopf algebras, I", Internat. J. Math. 10:4 (1999), 503-539. MR 2000c:16055 Zbl 0945.16033

[Drabant and van Daele 2001] B. Drabant and A. van Daele, "Pairing and quantum double of multiplier Hopf algebras", Algebr. Represent. Theory 4:2 (2001), 109-132. MR 2002d:16048 Zbl 0993.16024

[Drabant et al. 1999] B. Drabant, A. Van Daele, and Y. Zhang, "Actions of multiplier Hopf algebras", Comm. Algebra 27:9 (1999), 4117-4172. MR 2001d:16056 Zbl 0951.16013

[Elliott et al. 1988] G. A. Elliott, T. Natsume, and R. Nest, "Cyclic cohomology for one-parameter smooth crossed products”, Acta Math. 160:3-4 (1988), 285-305. MR 89h:46093 Zbl 0655.46054

[Hogbe-Nlend 1970] H. Hogbe-Nlend, "Complétion, tenseurs et nucléarité en bornologie", J. Math. Pures Appl. (9) 49 (1970), 193-288. MR 43 \#5279 Zbl 0199.18001

[Hogbe-Nlend 1977] H. Hogbe-Nlend, Bornologies and functional analysis, Notas de Matemática 62, North-Holland, Amsterdam, 1977. MR 58 \#17774 Zbl 0359.46004

[Kurose et al. 2000] H. Kurose, A. Van Daele, and Y. Zhang, "Corepresentation theory of multiplier Hopf algebras, II”, Internat. J. Math. 11:2 (2000), 233-278. MR 2001d:16060 Zbl 01629342

[Kustermans and Vaes 2000] J. Kustermans and S. Vaes, "Locally compact quantum groups", Ann. Sci. École Norm. Sup. (4) 33:6 (2000), 837-934. MR 2002f:46108 Zbl 1034.46508

[Kustermans and van Daele 1997] J. Kustermans and A. van Daele, " $C^{*}$-algebraic quantum groups arising from algebraic quantum groups”, Internat. J. Math. 8:8 (1997), 1067-1139. MR 99a:46130 Zbl 1009.46038

[Landstad and van Daele 2007] M. B. Landstad and A. van Daele, "Groups with compact open subgroups and multiplier Hopf $*$-algebras", preprint, 2007. arXiv math/0701525

[Meyer 1999] R. Meyer, Analytic cyclic homology, Ph.D. thesis, Universität Münster, 1999.

[Meyer 2004a] R. Meyer, "Bornological versus topological analysis in metrizable spaces", pp. 249278 in Banach algebras and their applications, Contemp. Math. 363, Amer. Math. Soc., Providence, RI, 2004. MR 2005k:46007 Zbl 1081.46004 
[Meyer 2004b] R. Meyer, "Smooth group representations on bornological vector spaces", Bull. Sci. Math. 128:2 (2004), 127-166. MR 2005c:22013 Zbl 1037.22011

[Meyer 2006] R. Meyer, "Combable groups have group cohomology of polynomial growth", $Q . J$. Math. 57:2 (2006), 241-261. MR 2007g:20051 Zbl 05135199

[Montgomery 1993] S. Montgomery, Hopf algebras and their actions on rings, CBMS Regional Conference Series in Mathematics 82, American Math. Society, Providence, 1993. MR 94i:16019 Zbl 0793.16029

[Natsume and Nest 1994] T. Natsume and R. Nest, "The local structure of the cyclic cohomology of Heisenberg Lie groups", J. Funct. Anal. 119:2 (1994), 481-498. MR 95g:22012 Zbl 0835.19003

[Rieffel 1993a] M. Rieffel, Deformation quantization for actions of $\mathbb{R}^{d}$, Memoirs Amer. Math. Soc. 506, American Math. Society, Providence, 1993. MR 94d:46072 Zbl 0798.46053

[Rieffel 1993b] M. A. Rieffel, "Compact quantum groups associated with toral subgroups", pp. 465-491 in Representation theory of groups and algebras, Contemp. Math. 145, Amer. Math. Soc., Providence, RI, 1993. MR 94i:22022 Zbl 0795.17017

[Rieffel 1995] M. A. Rieffel, "Non-compact quantum groups associated with abelian subgroups", Comm. Math. Phys. 171:1 (1995), 181-201. MR 96g:46066 Zbl 0857.17014

[Voigt 2007] C. Voigt, "Equivariant periodic cyclic homology", J. Inst. Math. Jussieu 6:4 (2007), 689-763. MR 2337312 Zbl 05195938

[Woronowicz and Pusz 1999] S. L. Woronowicz and W. Pusz, "Analysis on the quantum plane: a step towards Schwartz space for the $E_{q}(2)$ group", notes from work in progress, 1999, Available at http://www.fuw.edu.pl/ psoltan/prace/rgdr1999.pdf.

Received August 9, 2007. Revised October 4, 2007.

\section{CHRISTIAN VOIGT}

UNIVERSITÄT MÜNSTER

MATHEMATISCHES INSTITUT

EINSTEINSTRASSE 62

48149 MÜNSTER

GERMANY

cvoigt@math.uni-muenster.de 